\title{
Comparative Exploration of the Structure-Activity Space of Cloned $\alpha$-Like Octopamine Receptors from a Marine and a Terrestrial Arthropod $\$$
}

\author{
Dhwanil A. Dalwadi and John A. Schetz
}

\begin{abstract}
Department of Pharmacology and Neuroscience (D.A.D., J.A.S.), Graduate School of Biomedical Sciences, Institute for Healthy Aging, Center for Neuroscience Discovery, and Department of Medical Education (J.A.S.), Texas College of Osteopathic Medicine, University of North Texas Health Science Center, Fort Worth, Texas
\end{abstract}

Received January 23, 2017; accepted June 26, 2017

\section{ABSTRACT}

The $\alpha$-like octopamine receptors (OctR) are believed to be the evolutionary precursor to the vertebrate $\alpha_{2}$-adrenergic receptors $\left(\alpha_{2}\right.$-ARs) based upon sequence similarity and the ability to interact with norepinephrine and a number of compounds that bind with high affinity to $\alpha_{2}$-ARs. Barnacles and fruit flies are two prominent model marine and terrestrial representatives of the Arthropoda phylum, and although $\alpha$-like OctRs have been cloned from Balanus improvisus (BiOctR) and Drosophila melanogaster (DmOctR), little is known about the structureactivity space for these important species. A diverse panel of 22 probes spanning different structural classes were employed to interrogate the structure-activity of the BiOctR and DmOctR. While BiOctR and DmOctR exhibited similar functional profiles for mammalian biogenic amine G protein-coupled receptor agonists and antagonists, some ligands had dramatically different mechanisms of action. For instance, significant differences in the efficacy for some agonists were observed, including that vertebrate biogenic amines structurally related to octopamine acted as superagonists at the DmOctR but partial agonists at the BiOctR, and the two species diverged in their sensitivities to the $\alpha_{2}$-AR antagonist $\left[{ }^{3} \mathrm{H}\right]$ rauwolscine. Furthermore, sodium enhanced $\left[{ }^{3} \mathrm{H}\right]$ rauwolscine's interactions with the BiOctR, but not at a vertebrate $\alpha_{2}-\mathrm{AR}$. Molecular mechanistic studies indicate that rauwolscine interacts with the BiOctR, DmOctR, and $\alpha_{2 \mathrm{C}}$-adrenergic receptor at an allosteric site. In addition, compounds that acted as agonists at cloned $\alpha$-like BiOctR also induced a hyperactivity response in Balanus cyprids mediated by the $\alpha$-like OctR, suggesting that the receptor may serve as a higher throughput proxy for discovering compounds with potential cyprid deterrent properties.

\section{Introduction}

First discovered in the salivary gland of the octopus (Erspamer and Boretti, 1951), octopamine has since been found in high concentrations in excitable tissues of many arthropod species (Roeder, 1999). Given the close structural relationship between octopamine and norepinephrine (NE), it is perhaps not surprising that octopamine receptors (OctR) bear a close structural resemblance to the vertebrate receptors for NE. This has led to the notion that vertebrate $\alpha_{2}$ adrenergic receptors $\left(\alpha_{2}\right.$-AR) evolved from the invertebrate $\alpha$-like OctR, an idea reinforced by a pharmacological likeness (Dudai and Zvi, 1984; Nathanson, 1985; Roeder, 1995; Dahlström and Elwing, 2006; Lind et al., 2010).

This work was supported in part by grants from the Office of Naval Research [Grants N00014-08-1-0094 and N00014-12-M-0062]; the National Science Foundation [Grant 1248681]; a contract from Animal Biotech LLC, Dallas, Texas, USA; and institutional funds G67673.

https://doi.org/10.1124/mol.117.108456.

S This article has supplemental material available at molpharm. aspetjournals.org.
Because OctRs are found only in invertebrates and modulate a variety of processes, they are considered an attractive target for developing arthropod deterrents. Prior to the cloning of the OctR from the barnacle Balanus improvisus (BiOctR), the $\alpha$-adrenergic agonists medetomidine, clonidine, guanabenz, moxonidine, and tetrahydrozoline were shown to be capable of inhibiting cyprid settlement, as were certain antagonists, like phentolamine and idazoxan, although at much higher concentrations than the agonists (Dahlström et al., 2000; Dahms et al., 2004; Dahlström and Elwing, 2006). Despite being an $\alpha$-adrenergic receptor antagonist and being capable of antagonizing the cyprid settlementinhibiting effects of the agonist medetomidine, atipamezole alone was shown to have no effect on cyprid settlement (Dahlström et al., 2000). More recently, medetomidine was shown to act as an agonist at the cloned BiOctR and to induce hyperactivity in $B$. improvisus cyprids, and both effects were reversed by atipamezole (Lind et al., 2010). Yet beyond this, little is known about the pharmacology for BiOctR, and even less for Drosophila melanogaster OctR (DmOctR), prompting us to systematically interrogate the

ABBREVIATIONS: $\alpha_{2}$-AR, $\alpha_{2}$-adrenergic receptor; $\alpha_{2 C}$-AR, $\alpha_{2 C}$-adrenergic receptor; BiOctR, Balanus improvisus OctR; $\mathrm{B}_{\text {max }}$, receptor density; DmOctR, Drosophila melanogaster OctR; DMSO, dimethyl sulfoxide; EPI, epinephrine; FLIPR, fluorescence imaging plate reader; GPCR, G proteincoupled receptor; HBSS, Hank's buffered saline solution; $\mathrm{K}_{\mathrm{B}}$, antagonist dissociation constant; $\mathrm{K}_{\mathrm{D}}$, dissociation constant; $\mathrm{K}_{\mathrm{i}}$, dissociation constant determined from competition assays; NE, norepinephrine; NMDG, N-methyl-D-glucamine; OctR, octopamine receptor; ppt, parts per thousand. 
structure-activity space of cloned $\alpha$-like OctRs from Balanus and Drosophila.

\section{Materials and Methods}

Chemicals. The radioligand methyl (1S,15S,18S,19S,20S)-18-hydroxy1,3,11,12,14,15,16,17,18,19,20,21-dodecahydroyohimban-19-carboxylate $\left(\left[{ }^{3} \mathrm{H}\right]\right.$ rauwolscine, NET722250UC, $\left.80.5 \mathrm{Ci} / \mathrm{mmol}\right)$ was purchased from Perkin Elmer (Saint Louis, MO). Unless otherwise noted, all other drugs and reagents were purchased from Tocris Biosciences (via R\&D Systems, Minneapolis, MN), Santa Cruz Biotechnology (Dallas, TX), or SigmaAldrich (St. Louis, MO). All compounds except for octopamine were solubilized in dimethyl sulfoxide (DMSO) at concentrations ranging from 10 to $1000 \mathrm{mM}$ and diluted at least 1:1000 v/v in the final assay solution. Geneticin (G418) was purchased from Invivogen (San Diego, CA). Coral Life brand marine salts (CD-76412) were purchased from Doctors Foster and Smith (Rhinelander, WI).

Phylogenetic Analysis of OctRs. To assess the homology between OctR protein sequences, a phylogenetic tree was constructed using the maximum likelihood method (Guindon and Gascuel, 2003; Dereeper et al., 2008). In addition to $\alpha$-like and $\beta$-like OctRs from $B$. improvisus and D. melanogaster, included in the analysis were protein sequences for the human $\alpha_{2 \mathrm{C}}$-adrenergic receptor $\left(\alpha_{2 \mathrm{C}}-\mathrm{AR}\right)$ and several other cloned OctRs that have been functionally expressed and whose pharmacological properties are consistent with an $\alpha$-like or $\beta$-like OctR. The protein sequences were downloaded from National Center for Biotechnology Information and analyzed using the Phylogeny.fr online software. The multiple sequence alignment was performed using T-Coffee's default parameters. The accession numbers for the receptor sequences are ADI56271.1 (BiOctR or BiOct $\alpha-R 0$ ), ADI56275.1 (BiOct $\beta$-R4), ADI56274.1 (BiOct $\beta$-R3), ADI56273.1 (BiOct $\beta$-R2), ADI56272.1 (BiOct $\beta$-R1), AAC17442.1 (OAMB or DmOctR), Q9VCZ3.1 (Oct $\beta 1 R$ ), Q4LBB9.2 (Oct $\beta 2 R$ ), Q4LBB6.4 (Oct $\beta 3 R), N P \_000674.2\left(\alpha_{2 \mathrm{C}}-\mathrm{AR}\right), \mathrm{AAP} 93817.1$ (PaOctR or Pa oa1), and AAC61296.1 (LsOctR or Lym oa1).

Creation of a Stable Cell Line Expressing Either Barnacle or Fly Octopamine Receptors (BiOctR and DmOctR, Respectively). HEK293 cells, which are devoid of adrenergic receptors, were used for expressing the exogenous BiOctR and DmOctR. The BiOctR (accession GU074418) expression vector was a gift of
U. Lind (Lind et al., 2010), and DmOctR (accession AF065443) vector was a gift of R. Davis (Han et al., 1998). Both expression vectors had the pcDNA3.1 backbone and were under the control of the cytomegalovirus promoter. Twenty micrograms of the expression vectors were transfected into HEK293 cells followed by G418 selection as described previously (Ericksen et al., 2009). Individual colonies were evaluated for receptor expression by measuring octopamine-mediated changes in intracellular calcium. The clone that produced the largest response was used for subsequent functional and radioligand-binding experiments.

Measurement of Affinities and Densities of Receptors Using Radioligand-Binding Assays. HEK293 cells stably expressing BiOctR, DmOctR, or the human $\alpha_{2 \mathrm{C}}-\mathrm{AR}$ (accession NP_000674.2) (stable cell line expressing the $\alpha_{2 \mathrm{C}} \mathrm{AR}$ was a gift of M. Toews) were harvested from culture flasks using $\mathrm{Ca}^{2+}$ - and $\mathrm{Mg}^{2+}$-free Dulbecco's phosphate-buffered saline (55-031-PB; Fisher Scientific, Pittsburgh, PA) supplemented with $5 \mathrm{mM}$ EDTA (E6511; Sigma-Aldrich, St. Louis, MO). Membranes expressing the receptor of interest were harvested, as described previously (Ericksen et al., 2009). All binding studies were performed in $1 \mathrm{ml}$ volume using $\left[{ }^{3} \mathrm{H}\right]$ rauwolscine as the radioligand. To optimize the binding reaction conditions, the following conditions were tested: $50 \mathrm{mM}$ Tris- $\mathrm{HCl}, \mathrm{pH} 7.4$, at $25^{\circ} \mathrm{C}$, supplemented with either $15,50,120,240,360$, or $480 \mathrm{mM} \mathrm{NaCl}$ or $480 \mathrm{mM}$ $\mathrm{NaCl}$ plus either $10.5 \mathrm{mM} \mathrm{KCl}$ or $10.5 \mathrm{mM} \mathrm{KCl}$ plus $53.8 \mathrm{mM} \mathrm{MgCl}_{2}$. Other buffer test conditions included 35 parts per thousand (ppt) marine salt, which is a scientific grade salt preparation that replicates natural sea water chemistry, and $50 \mathrm{mM}$ potassium phosphate buffer at $\mathrm{pH}$ 7.4. The optimal binding buffer, determined to be $50 \mathrm{mM}$ Tris$\mathrm{HCl}, 240 \mathrm{mM} \mathrm{NaCl}, \mathrm{pH} 7.4$, at $25^{\circ} \mathrm{C}$, was used for all subsequent radioligand experiments, except when sodium concentration was a test variable, other concentrations of sodium were used.

The expression levels [receptor density $\left(\mathrm{B}_{\max }\right)$ ] for BiOctR and $\alpha_{2 \mathrm{C}}-\mathrm{AR}$ and the dissociation constant $\left(\mathrm{K}_{\mathrm{D}}\right)$ of $\left[{ }^{3} \mathrm{H}\right]$ rauwolscine were determined by saturation isotherm binding using the rapid filtration technique, as described previously (Kortagere et al., 2004). Eight concentrations of $\left[{ }^{3} \mathrm{H}\right]$ rauwolscine ranging from 0.0625 to $8 \mathrm{nM}$ were tested. Nonspecific binding was defined as binding in the presence of $10 \mu \mathrm{M}$ atipamezole. Membrane protein concentrations varied from 0.02 to $0.06 \mathrm{mg} / \mathrm{ml}$. Competition binding was used to measure the affinity [dissociation constant determined from competition assays $\left(\mathrm{K}_{\mathrm{i}}\right)$ ] of ligands, in which the concentration of the test ligand was increased and the concentration

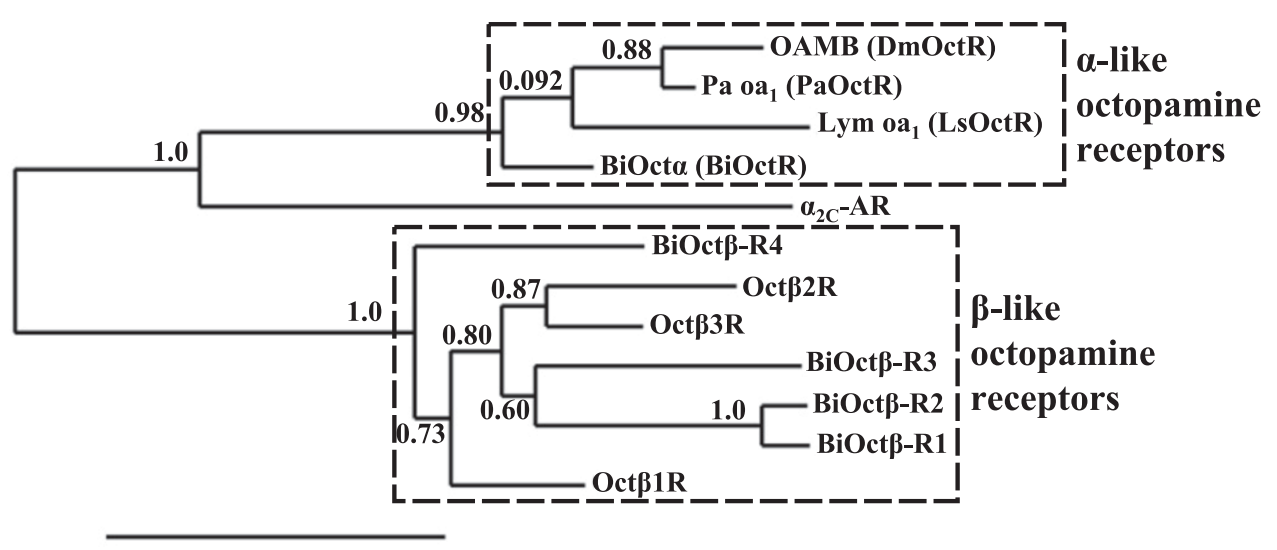

0.4

Fig. 1. On the basis of sequence similarity, the barnacle OctR is more distantly related to the fly than the cockroach OctR. Protein sequences for $\alpha$-like and $\beta$-like OctRs were compared with the human $\alpha_{2 \mathrm{C}}$-AR by phylogenetic tree analysis. Only OctR sequences having undergone extensive functional and pharmacological characterization were included in the analysis. This included receptor sequences from the barnacle B. improvisus: an $\alpha$-like ADI56271.1 (BiOctR or BiOct $\alpha$ ), and four $\beta$-like ADI56275.1 (BiOct $\beta$-R4), ADI56274.1 (BiOct $\beta$-R3), ADI56273.1 (BiOct $\beta$-R2), ADI56272.1 (BiOct $\beta$-R1) OctRs; from the fruit fly D. melanogaster: an $\alpha$-like AAC17442.1 (OAMB or DmOctR), and three $\beta$-like Q9VCZ3.1 (Oct $\beta 1 \mathrm{R}$ ), Q4LBB9.2 (Oct $\beta 2 R$ ), Q4LBB6.4 (Oct $\beta 3 R$ ) OctRs; an $\alpha$-like OctR from an aquatic snail [AAC61296.1 (Lym oa1 or LsOctR)], and an $\alpha$-like OctR from a terrestrial arthropod (cockroach) [AAP93817.1 (Pa oa 1 or PaOctR)]. BiOctR shares $\leq 32 \%$ sequence similarity with the human $\alpha_{2 \mathrm{C}}$-AR and the $\beta$-like OctRs, but $\geq 43 \%$ sequence similarity with the $\alpha$-like OctRs. Of the two $\alpha$-like OctRs from terrestrial arthropods, BiOctR shares $51 \%$ sequence similarity to PaOctR and $46 \%$ to DmOctR. Branch lengths represent change over time, and the longer the branch, the greater the amount of change. The number on each branch is a measure of support for the node; the higher the number, the greater the probability that the groups in the node cluster together. 
A

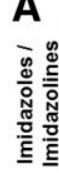

פ

Atipamezole $+10 \mathrm{nM}$ Levomedetomidine Atipamezole $+1 \mathrm{nM}$ Dexmedetomidine $1 \mathrm{nM}$ Dexmedetomidine
Atipamezole $+1 \mu \mathrm{M}$ Tizanidine Atipamezole $+11 \mu$ M Tizanidine Atipamezole $+1 \mu$ M Naphazoline Atipamezole $+1 \mu \mathrm{M}$ Clonidine

$100 \mu$ M 5-HT

을 Atipamezole $+100 \mu \mathrm{M}(+)$-Norepinephrine Atipamezole $+1,1 \mathrm{MM}(+)-$-Norepinephrine ${ }_{1}^{\mu M} \mathrm{M}(-)-$ Norepinephrine Atipamezole $+1 \mu \mathrm{M}$ (-)-Epinephrine Atipamezole $+1 \mu \mathrm{M}$ M Octopamine $\mu$ M Octopamine
Atipamezole Vehicle
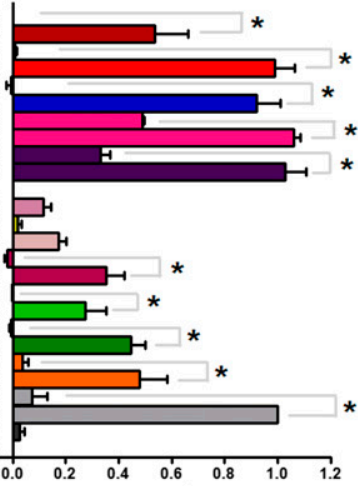
Change in $\left[\mathrm{Ca}^{2+}\right]_{\text {intracellular }}$

C

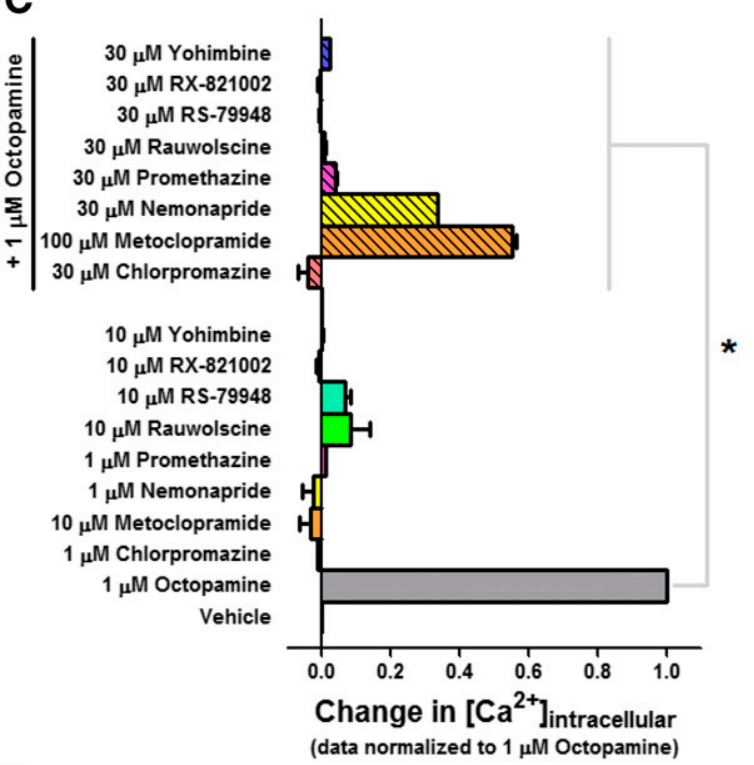

E

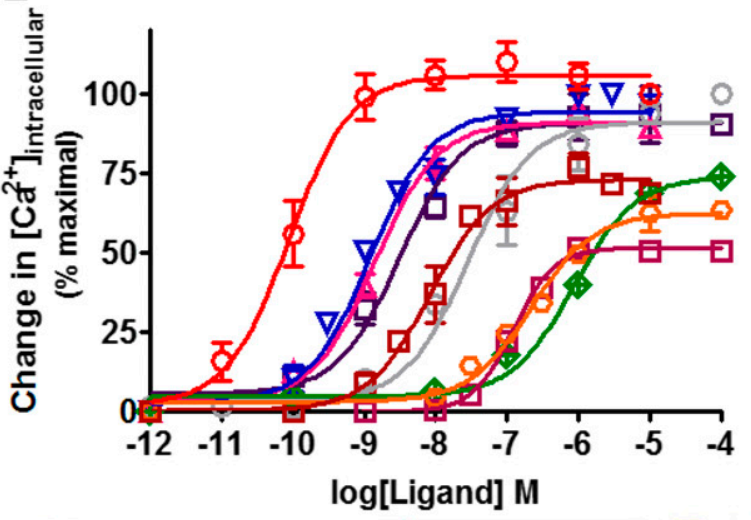

B

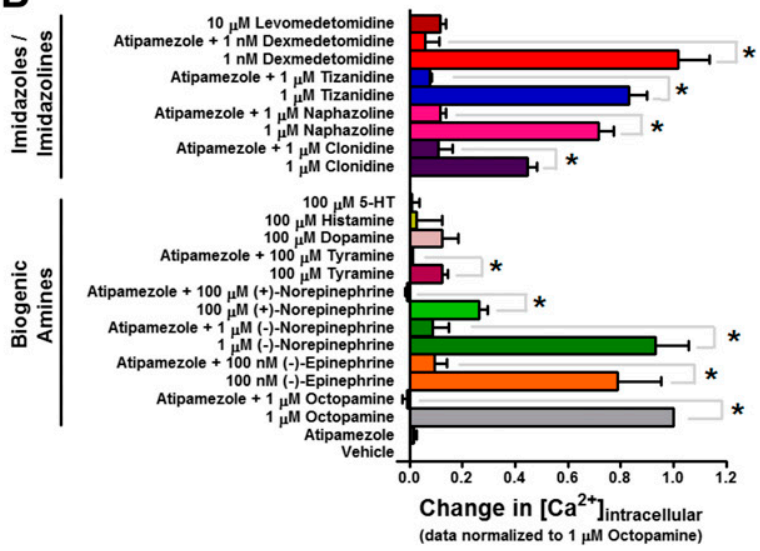

D

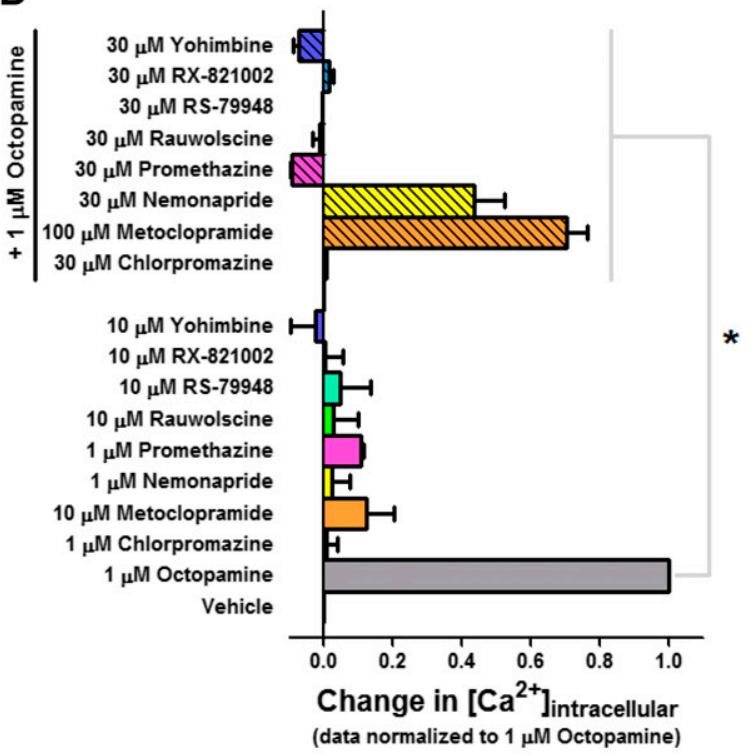

$\mathbf{F}$

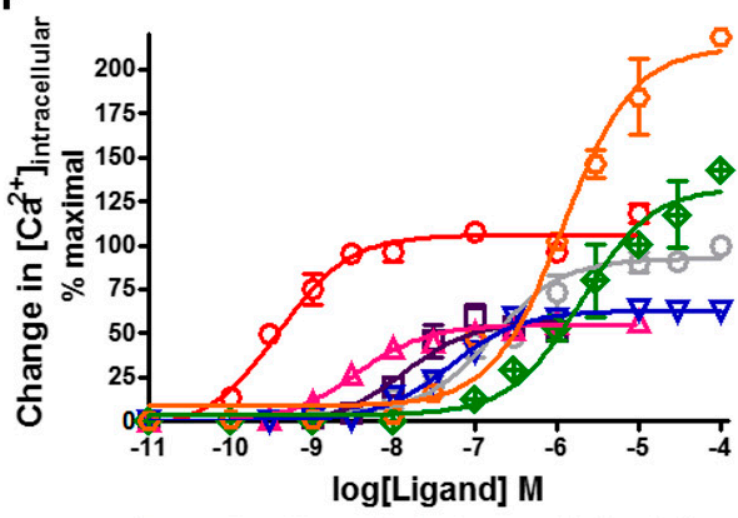

Clonidine $\square$ Levomedetomidine

\section{Dexmedetomidine \\ Naphazoline \\ Tizanidine \\ (-)-Epinephrine \\ (-)-Norepinephrine}

Fig. 2. Mammalian GPCR agonists and antagonists have similar functional responses and agonist rank order potencies at the barnacle and fly OctRs. Changes in intracellular calcium were used to measure the activation of the $\mathrm{G}_{\mathrm{q}}$-coupled $\alpha$-like BiOctR and DmOctR stably transfected in HEK293 cells. The response data for each agonist are normalized to the response generated by $1 \mu \mathrm{M}$ of the endogenous full agonist octopamine. The actions of ligands generating significant calcium responses were reversed with $30 \mu \mathrm{M}$ antagonist atipamezole, and the antagonist effect of ligands were tested by their ability to reverse the $1 \mu \mathrm{M}$ octopamine response. (A and B) Functional profile of mammalian GPCR agonists for the BiOctR and the DmOctR, respectively. Imidazoles = dexmedetomidine and levomedetomidine. Imidazolines = clonidine, naphazoline, and tizanidine. (C and D) Functional effects of mammalian GPCR antagonists at the BiOctR and DmOctR, respectively. Averaged data for all experiments are plotted as means \pm S.E.M. One-way analysis of variance, followed by Bonferroni's post hoc, was performed to evaluate statistical significance for all groups shown within each panel. $* P<0.05$ for reversal of agonist responses by the antagonists. (E and F) Agonist concentration-response curves for the BiOctR and DmOctR, respectively. Averaged data for all experiments are plotted as mean \pm S.E.M. from two or more independent experiments. The corresponding potencies and efficacy values are listed in Table 1. See Supplemental Table 1 for chemical structures, and Supplemental Tables 2 and 3 for numerical values for the bar graphs shown in (A-D). 
of $\left[{ }^{3} \mathrm{H}\right]$ rauwolscine was held constant at $2 \mathrm{nM}$. Equilibrium inhibition constants $\left(\mathrm{K}_{\mathrm{i}}\right)$ representing binding affinities were calculated from $\mathrm{IC}_{50}$ values using the Cheng-Prusoff equation: $\mathrm{K}_{\mathrm{i}}=\mathrm{IC}_{50}\left(1+[\right.$ ligand $\left.] / \mathrm{K}_{\mathrm{D}}\right)$. Concentration-response curves were fitted with a four-parameter logistics equation that included a variable slope using a 95\% confidence interval for all curve fits using GraphPad Prism version 5.0 (San Diego, CA).

Assessment of Sodium Sensitivity of BiOctR via Sodium Replacement. To assess whether the sodium sensitivity exhibited by $\left[{ }^{3} \mathrm{H}\right]$ rauwolscine is due to sodium and not charge, the $240 \mathrm{mM}$ in the binding buffer was replaced with $240 \mathrm{mM}$ N-methyl-D-glucamine (NMDG; M-2004; Sigma Aldrich), which is a commonly used sodium substitute as it has a +1 charge, but does not bind the sodium site of $\mathrm{G}$ protein-coupled receptors (GPCR). To determine whether the sodium site is accessible from the intracellular or extracellular side, whole-cell binding was performed. The whole-cell pellet was resuspended in a modified Hank's buffered saline solution (HBSS) buffer containing either $\mathrm{NaCl}$ or NMDG, and these intact cells were used for radioligand binding, as described previously, except HBSS with either $\mathrm{NaCl}$ or NMDG was used as the binding buffer instead of the Tris-HCl buffer. The composition of the HBSS buffer was the same as the commercially available Gibco HBSS (cat. no. 14065; Thermo Fisher Scientific, Waltham, MA), except sodium phosphate dibasic was replaced with potassium phosphate dibasic and supplemented with either $140 \mathrm{mM} \mathrm{NaCl}$ or $140 \mathrm{mM}$ NMDG.

Protein Assay. Membrane protein concentrations were measured by bicinchoninic acid assay (Life Technologies, Grand Island, NY), according to the manufacturer's instructions. Protein standard curves were constructed using purified bovine serum albumin that comes with the kit.

Intracellular Calcium Assay: Measurement of $\mathbf{G}_{\mathbf{q}}$-Coupled Responses. HEK293 cells stably expressing the BiOctR or DmOctR receptors were used to evaluate change in intracellular calcium as a measure of receptor activation using the FLIPR calcium-6 QF dye (Molecular Devices, Sunnyvale, CA), as described previously (Dalwadi et al., 2016). Following baseline subtraction of basal levels of intracellular calcium in the absence of any receptor stimulation, all data were normalized to the maximal functional response produced by a saturating concentration of the endogenous full agonist octopamine defined as the upper asymptote of the sigmoidal curve.

Interaction of Phenoxybenzamine and Rauwolscine at the BiOctR. To assess the reversible or irreversible nature of interaction of phenoxybenzamine and rauwolscine, 1 million BiOctR-expressing cells were plated in T-25 flasks. The cells were treated with either vehicle (1:1000 DMSO, v/v), $10 \mu \mathrm{M}$ phenoxybenzamine, or $10 \mu \mathrm{M}$ rauwolscine for 12 hours. Cells were then washed three times with Dulbecco's phosphate-buffered saline, and each wash was for 30 minutes. Following the last wash step, cell membranes were harvested and prepared for radioligand binding, as described above.

Operational Model of Agonism and RauwolscineDexmedetomidine Functional Competition Assay. HEK293 cells stably expressing the BiOctR receptors were plated in clearbottom, black-walled, 96-well plates, as described above. On the day of the experiment, media was removed and cells were treated with increasing concentrations of the irreversible antagonist phenoxybenzamine (for operational model) or the reversible antagonist rauwolscine (for Schild shift) prepared in $20 \mathrm{mM}$ HEPES Hank's buffer, pH 7.4. Immediately after adding the antagonists, FLIPR calcium- 6 QF dye was added and incubated at $37^{\circ} \mathrm{C}$ for 3 hours in the dark. Following dye loading, fluorescent signals were measured, as described above. For each concentration of the antagonist, a full concentration-response curve of the agonist was generated. All data sets were normalized to the maximum response obtained in the absence of the antagonists. For calculating the dissociation constant $\mathrm{K}_{\mathrm{A}}$ for the agonists dexmedetomidine and octopamine, phenoxybenzamine-agonist concentration responses were curve-fitted using the operational model of agonism (Leff et al., 1985; Black et al., 2010) in Prism 5.0 (operational model-depletion equation). For the rauwolscine-dexmedetomidine functional competition assay, rauwolscine-dexmedetomidine concentration-response curves were fitted with a four-parameter logistic equation that included a variable slope. If the results met all assumptions of Schild analysis (i.e., the antagonist is purely competitive rather than allosteric and does not result in reduction of efficacy), the data will be linear when transformed into a Schild plot, and from such a plot the antagonist potency [ $\mathrm{pA}_{2}$ or $\mathrm{K}_{\mathrm{B}}$ (antagonist dissociation constant)] can be obtained as the point at which the line crosses the $x$-axis.

Cyprid Motility Assay. Cyprids of Balanus amphitrite were provided by D. Rittschof (Duke Marine Laboratory, Beaufort, NC) using methods described previously (Berntsson et al., 2000). Cyprid motility was assessed by trapping the cyprids in an agarose matrix using a procedure described by Lind et al. (2010) with the following modifications. Briefly, 2\% ultra-low gelling agarose (A-2576; SigmaAldrich) was prepared using $35 \mathrm{ppt}$ marine salt. The agarose was put into solution by heating in boiling salt water, and then the agarose solution was allowed to cool to just above its gelling temperature by incubating in a $28^{\circ} \mathrm{C}$ water bath. Once cooled, DMSO vehicle or test compounds (e.g., dexmedetomidine, levomedetomidine, clonidine, naphazoline, tizanidine, octopamine) or test compounds plus $50 \mu \mathrm{M}$

TABLE 1

Potency and efficacy of ligands functioning as BiOctR and DmOctR agonists

Changes in intracellular calcium were used as a measure of receptor activation and for generating agonist concentrationresponse curves. The curves were fitted with a four-parameter logistic equation to obtain potency $\left(\mathrm{EC}_{50}\right)$ values, which are presented as means \pm S.E.M. Efficacies $(\alpha)$ significantly less than octopamine are denoted by an asterisk, whereas those significantly greater are denoted by a dagger $(P<0.05$, analysis of variance followed by a Bonferroni's post hoc).

\begin{tabular}{lccccc}
\hline \multirow{2}{*}{ Ligand } & \multicolumn{2}{c}{ BiOctR } & & \multicolumn{2}{c}{ DmOctR } \\
\cline { 2 - 3 } \cline { 5 - 6 } & $\begin{array}{c}\text { Efficacy } \\
(\alpha \pm \text { S.E.M. })\end{array}$ & $\begin{array}{c}\mathrm{EC}_{50} \\
(\mathrm{nM} \pm \text { S.E.M. })\end{array}$ & & $\begin{array}{c}\text { Efficacy } \\
(\alpha \pm \text { S.E.M. })\end{array}$ & $\begin{array}{c}\mathrm{EC}_{50} \\
(\mathrm{nM} \pm \mathrm{S} . \mathrm{E} . \mathrm{M} .)\end{array}$ \\
\hline Dexmedetomidine & $0.99 \pm 0.10$ & $0.086 \pm 0.023$ & & $1.1 \pm 0.073$ & $0.39 \pm 0.11$ \\
Tizanidine & $0.97 \pm 0.091$ & $1.2 \pm 0.32$ & & $0.64 \pm 0.039^{*}$ & $49 \pm 6.1$ \\
Naphazoline & $0.89 \pm 0.11$ & $1.4 \pm 0.25$ & & $0.57 \pm 0.052^{*}$ & $3.5 \pm 0.53$ \\
Clonidine & $0.90 \pm 0.029$ & $3.1 \pm 1.0$ & & $0.52 \pm 0.029^{*}$ & $13 \pm 4.7$ \\
Levomedetomidine & $0.69 \pm 0.050^{*}$ & $8.3 \pm 2.2$ & & No activity & N.D.a \\
Octopamine & 1.0 & $28 \pm 8.5$ & & 1.0 & Very weak agonist \\
Tyramine & $0.50 \pm 0.050^{*}$ & $130 \pm 22$ & & $2.2 \pm 0.31^{\dagger}$ & N.D. \\
(-)-Epinephrine & $0.63 \pm 0.052^{*}$ & $220 \pm 46$ & & $1200 \pm 270$ \\
(-)-Norepinephrine & $0.74 \pm 0.049^{*}$ & $860 \pm 170$ & & $1.4 \pm 0.19^{\dagger}$ & $1900 \pm 530$ \\
(+)-Norepinephrine & Very weak agonist & N.D. & & Very weak agonist & N.D. \\
Dopamine & No activity & N.D. & & No activity & N.D. \\
Serotonin & No activity & N.D. & & No activity & N.D. \\
Histamine & No activity & N.D. & & No activity & N.D. \\
\hline
\end{tabular}

${ }^{a}$ Not determined.

${ }^{b} \mathrm{~A}<30 \%$ response compared with octopamine at $100 \mu \mathrm{M}$. 


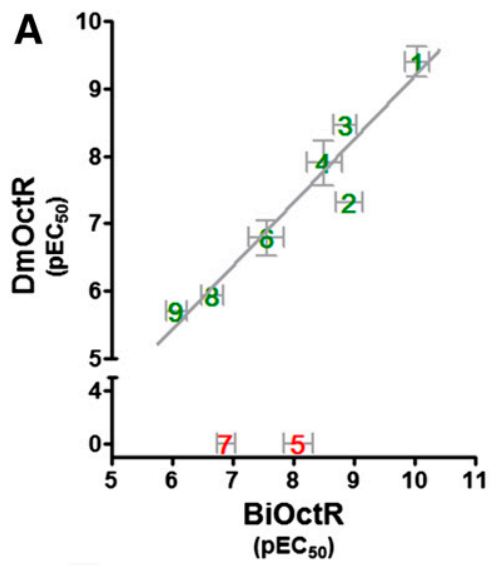

B

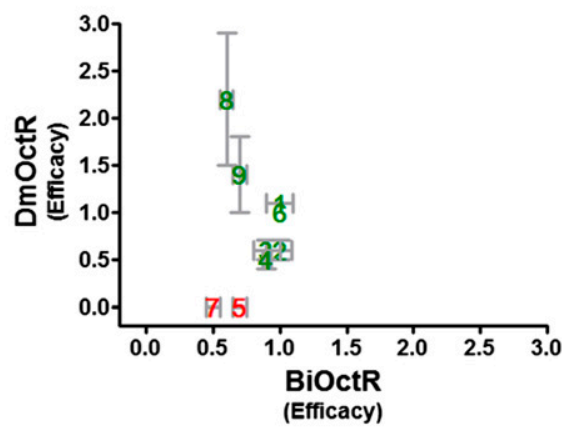

1 Dexmedetomidine 2 Tizanidine 3 Naphazoline 4 Clonidine 5 Levomedetomidine

6 Octopamine 7 Tyramine 8 (-)-Epinephrine 9 (-)-Norepinephrine

Fig. 3. Agonists of BiOctR and DmOctR share a similar potency profile. (A) The log of the potencies (pEC50 \pm S.E.M.) for biogenic amine and imidazole/imidazoline agonists at the BiOctR and DmOctR are plotted against one another to visualize the correlations. Tyramine and levomedetomidine were assigned an $\mathrm{EC}_{50}$ of 0 only for the completeness of the comparison, but were excluded from the correlational analysis. Analysis of the compound potencies between the two receptors indicates they are highly correlated with a Pearson's $r^{2}=0.91$, yet the potencies for all compounds were consistently lower at the fly than at the barnacle receptor, leading to a slope slightly more shallow than unity (slope $=0.92 \pm 0.13$ ). The largest difference in potencies was for tizanidine, which had an approximately 42 -fold higher affinity for the barnacle than the fly receptor. (B) For comparison, the efficacies are plotted as well, although the correlation is rather poor (Pearson's $r^{2}=0.63$ ), consistent with there being large differences in the efficacies for a number of the agonists, including the surprising finding that only at the fly receptor (-)-epinephrine and (-)-NE acted as superagonists, whereas all of the imidazoline agonists had partial agonist properties.

atipamezole were added to samples of the agarose and mixed by vortexing. In each case, the final DMSO concentration was 1:10,000 $\mathrm{v} / \mathrm{v}$. To wells of a 96 -well plate, three to five cyprids in $5 \mu \mathrm{l}$ seawater were added per well and then trapped by overlaying them with $200 \mu \mathrm{l}$ agarose mixtures containing either vehicle or test compounds. The agarose was then allowed to solidify at room temperature for 1 hour, after which the cyprids were viewed under a stereoscope at $3 \times$ magnification and individual cyprid movements were quantified as the number of forceful body movements per minute. Specifically, any abrupt and forceful flicking movement of the legs or dorsiflexion of the back was counted as a movement, although movements of the antennas, which were typically slower, were not.

A

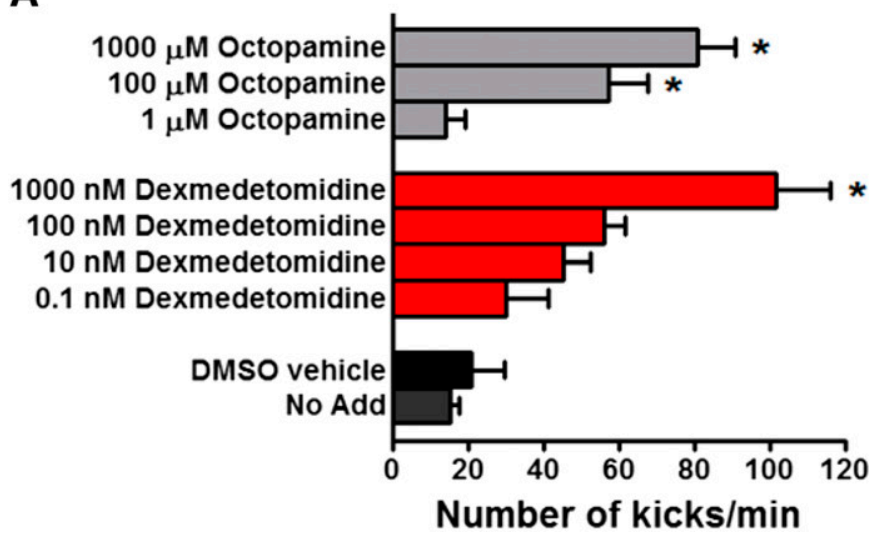

Statistical Analyses. Each data point for each molecular experiment was sampled in triplicate, and then each experiment was repeated two or more times. The triplicate measurements were averaged to yield the value for that single experiment: the resulting value was considered as $n=1$. For the cyprid motility assays, three to five cyprids were used in each group, and each experiment was repeated two to three times. The data for all similar experiments were averaged and plotted as means \pm S.E.M. To evaluate statistical significance, a one-way analysis of variance, followed by Bonferroni's post hoc analysis (for more than two groups) or a Student's $t$ test (for two groups) was performed, and $P<0.05$ was used as the cutoff for significance.

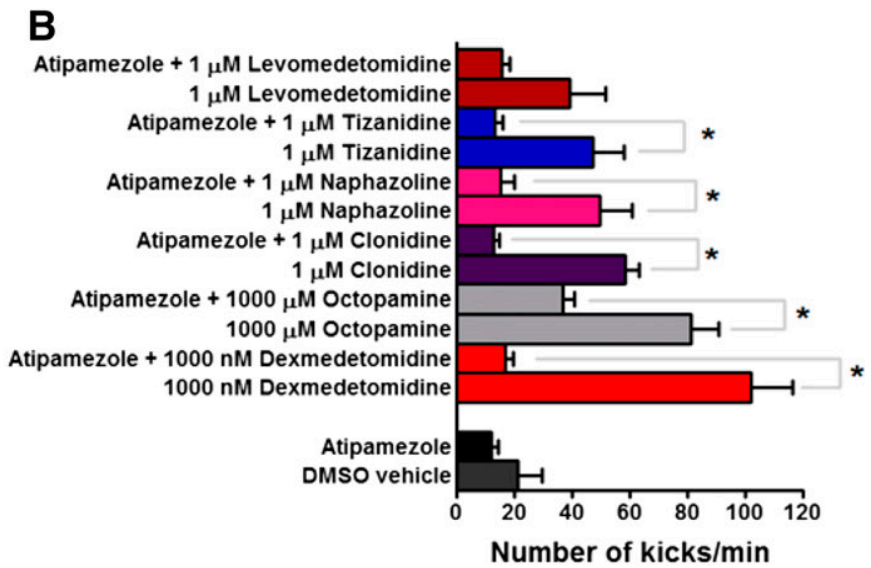

Fig. 4. OctR agonists induce hyperactivity in cyprids from B. amphitrite. Cyprid hyperactivity was measured by quantifying their body movements in agarose containing vehicle, test compound, or test compound plus $50 \mu \mathrm{M}$ atipamezole. (A) Dexmedetomidine and octopamine induced cyprid hyperactivity in a concentration-dependent manner. (B) Atipamezole $(50 \mu \mathrm{M})$ inhibits OctR agonist-mediated hyperactivity. Data presented as mean \pm S.E.M. from five or more independent experiments. One-way analysis of variance, followed by Bonferroni's post hoc, was performed to evaluate statistical significance. $* P<0.05$, (A) significantly greater response than vehicle (DMSO) and (B) significant inhibition of agonist response by atipamezole. See Supplemental Table 4 for numerical values for the bar graphs shown in (A) and (B). 


\section{Results}

The molecular mechanisms for a series of structurally diverse ligands acting on the cloned barnacle and fruit fly $\alpha$-like OctRs were explored to probe the structure-activity space of these receptors. This included an evaluation of steroselectivities and the sodium sensitivity of some ligands. The BiOctR exhibited sodium sensitivity for the antagonist rauwolscine, and the functional activity profile of the ligands bore a resemblance to the DmOctR, although in most cases the agonists had lower efficacy. An exception to this was for (-)-NE and (-)-epinephrine (EPI), which acted as partial agonists at BiOctR, but as superagonists at DmOctR. Unexpectedly, $\left[{ }^{3} \mathrm{H}\right]$ rauwolscine's affinity for the barnacle receptor was increased by sodium, although this was not the case for either the fly receptor or the human $\alpha_{2}$-AR.

Phylogenetic Analysis of OctRs. Protein sequences of four cloned $\alpha$-like OctRs, seven $\beta$-like OctRs, and the human $\alpha_{2 \mathrm{C}}$-AR were compared by phylogenetic analysis. As expected, the $\beta$-like OctRs clustered together and parsed away from the $\alpha$-like OctRs and the $\alpha_{2 \mathrm{C}}$-AR; similarly, the $\alpha$-like OctRs clustered together and parsed away from the $\alpha_{2 \mathrm{C}}-\mathrm{AR}$, indicating its more distant relationship (Fig. 1). Furthermore, when the BiOctR sequence was compared with the other three $\alpha$-like OctRs, the alignment scores for protein sequence identity were as follows: DmOctR $=46 ; \mathrm{PaOctR}=51$; LsOctR $=43$. All three $\alpha$-like OctRs had more than $40 \%$ sequence similarity to the BiOctR and share common $G_{q}$ signaling functions, indicating that they are orthologs. Both PaOctR and DmOctR are terrestrial arthropods, and because DmOctR parsed away from BiOctR more than PaOctR, the homologous nature of the two more distant OctRs was explored in greater detail by systematically comparing their pharmacological profiles.

Functional Pharmacology of BiOctR and DmOctR. Because vertebrate $\alpha_{2}$-AR are homologs of invertebrate OctRs, a series of adrenergic receptor ligands were used to evaluate cloned $\alpha$-like OctRs from barnacle and fly. Octopamine is the endogenous agonist for OctRs and served as a positive control for receptor activation and the cataloguing of efficacies of other agonists. All of the major biogenic amines were tested for their ability to activate barnacle and fly OctRs, except for acetylcholine, which was not tested because HEK293 cells contain
A

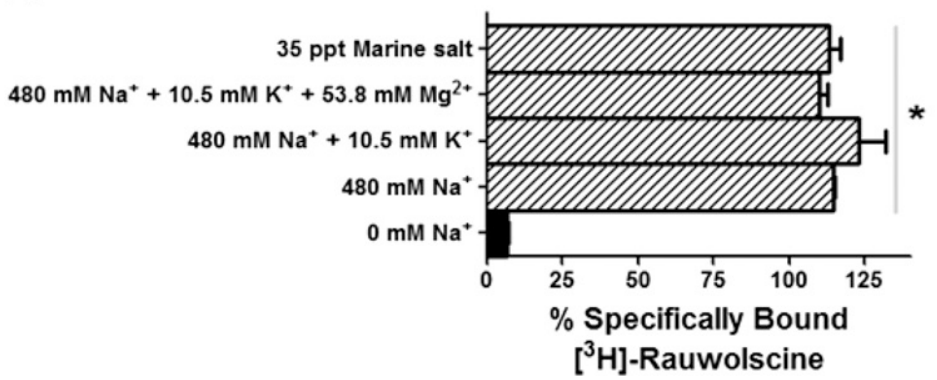

$\left[{ }^{3} \mathrm{H}\right]$-Rauwolscine
B

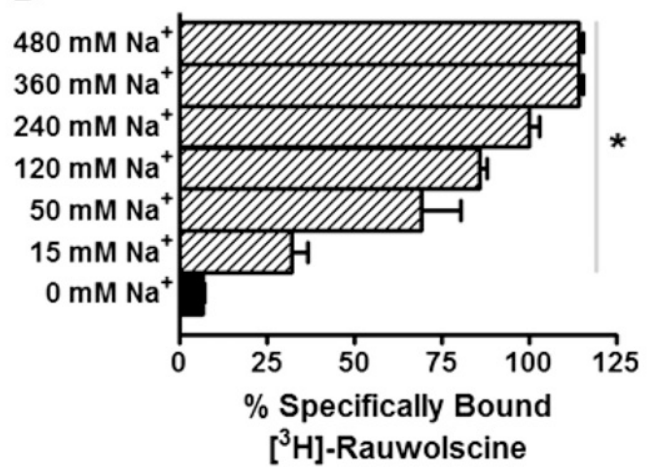

D

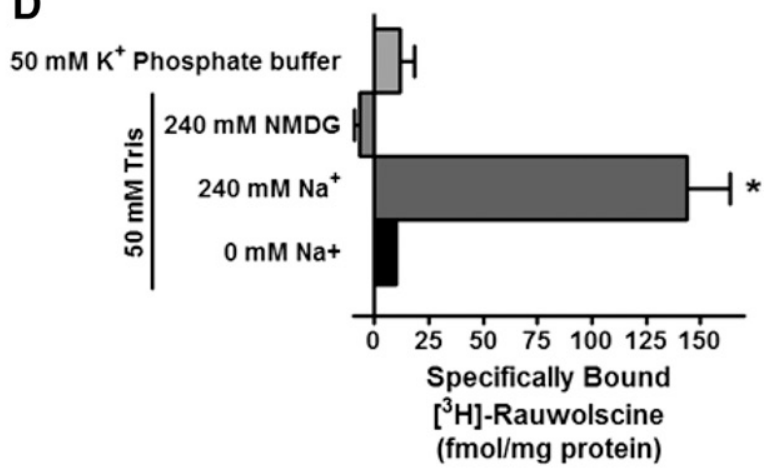

Fig. 5. $\left[{ }^{3} \mathrm{H}\right]$ Rauwolscine-specific binding to BiOctR can only be detected in the presence of a high concentration of sodium, and the sodium site is only accessible from the intracellular side. HEK293 cells expressing the BiOctR served as the membrane source for the radioligand-binding assay. Different salt conditions were tested to determine ideal binding conditions for $\left[{ }^{3} \mathrm{H}\right]$ rauwolscine. Whole-cell radioligand binding and membrane binding were performed to evaluate the accessibility of the sodium site and effect of ionic charge, respectively. The physiologic buffer HBSS with either NaCl or the sodium substitute NMDG served as the whole-cell binding assay buffer, and $50 \mathrm{mM}$ Tris buffer with either 240 sodium or $240 \mathrm{mM}$ NMDG was used for binding in broken membrane preparations. A $50 \mathrm{mM}$ potassium phosphate buffer was also tested in the membrane-binding assay as well. (A) The amount of specifically bound $\left[{ }^{3} \mathrm{H}\right]$ rauwolscine is dependent upon sodium concentration, and the presence of $\mathrm{K}^{+}$or $\mathrm{Mg}^{2+}$ does not further enhance specific binding. (B) Sodium increases levels of $\left[{ }^{3} \mathrm{H}\right]$ rauwolscine-specific binding in a concentration-dependent manner with no significant increase observed beyond $240 \mathrm{mM}$. (C) Whole-cell binding in the presence of either sodium or the same concentration of NMDG to maintain ionic charge and osmolarity demonstrated that extracellular sodium has no significant effect on $\left[{ }^{3} \mathrm{H}\right]$ rauwolscine-specific binding, $P>0.05$ by Student's $t$ test. (D) Radioligand binding using broken membrane preparations demonstrated that ionic charge had no effect on $\left[{ }^{3} \mathrm{H}\right]$ rauwolscine-specific binding. Data were plotted as the mean \pm S.E.M. from two or more independent experiments. One-way analysis of variance followed by Bonferroni's post hoc was performed to evaluate statistical significance. $* P<0.05$ compared with $0 \mathrm{mM}$ sodium. See Supplemental Tables 5 and 6 for numerical values for the bar graphs shown in (A-D). 
an endogenous $\mathrm{G}_{\mathrm{q}}$-coupled M3 muscarinic acetylcholine receptor (Dalwadi et al., 2016). Although it had the most favorable interactions with octopamine, the biogenic amines tyramine, (-)-EPI, (-)-NE, and (+)-NE also activated the $\mathrm{BiOctR}$, and the responses were reversed by the antagonist atipamezole (Fig. 2A, $P<0.05$ ), which by itself had no effect. Consistent with a previous report (Lind et al., 2010), even when tested at a very high concentration $(100 \mu \mathrm{M})$, the biogenic amines serotonin, histamine, and dopamine were unable to activate the BiOctR (Fig. $2 \mathrm{~A}, P>0.05$ ). The $\alpha$-adrenergic receptor agonists clonidine, naphazoline, tizanidine, dexmedetomidine, and levomedetomidine each stimulated the BiOctR, and their responses were all reversed by atipamezole (Fig. 2A, $P<0.05$ ). The functional profile for DmOctR was similar, except that levomedetomidine was unable to significantly activate the receptor at $10 \mu \mathrm{M}$ (Fig. $2 \mathrm{~B}, P>0.05)$. Like at the BiOctR, serotonin, histamine, and dopamine were unable to activate the DmOctR as well (Fig. 2B, $P>0.05$ ).

In addition to agonists, the functional properties for different structural classes of mammalian GPCR antagonists were also evaluated at BiOctR and DmOctR. Although all of these ligands failed to mediate activation of the $G_{q}$ pathway via BiOctR and DmOctR, each was able to significantly inhibit activation of these receptors by octopamine (Fig. 2, C and D, $P<0.05)$. The functional profile for all of the antagonists
A

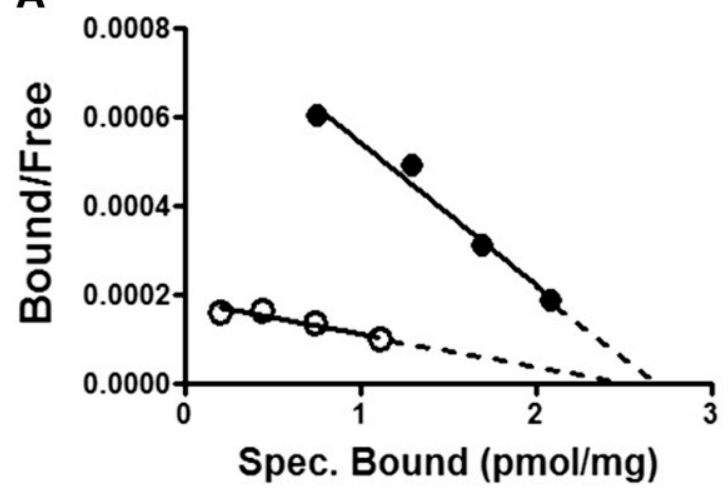

- $15 \mathrm{mM} \mathrm{Na}^{+} \quad 240 \mathrm{mM} \mathrm{Na}^{+}$
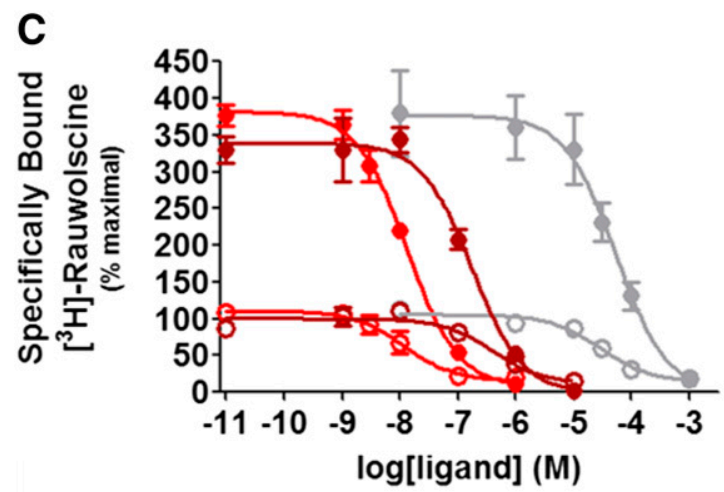

- Dexmedetomidine

- Octopamine

- Levomedetomidine

- $15 \mathrm{mM} \mathrm{Na}^{+}$
B
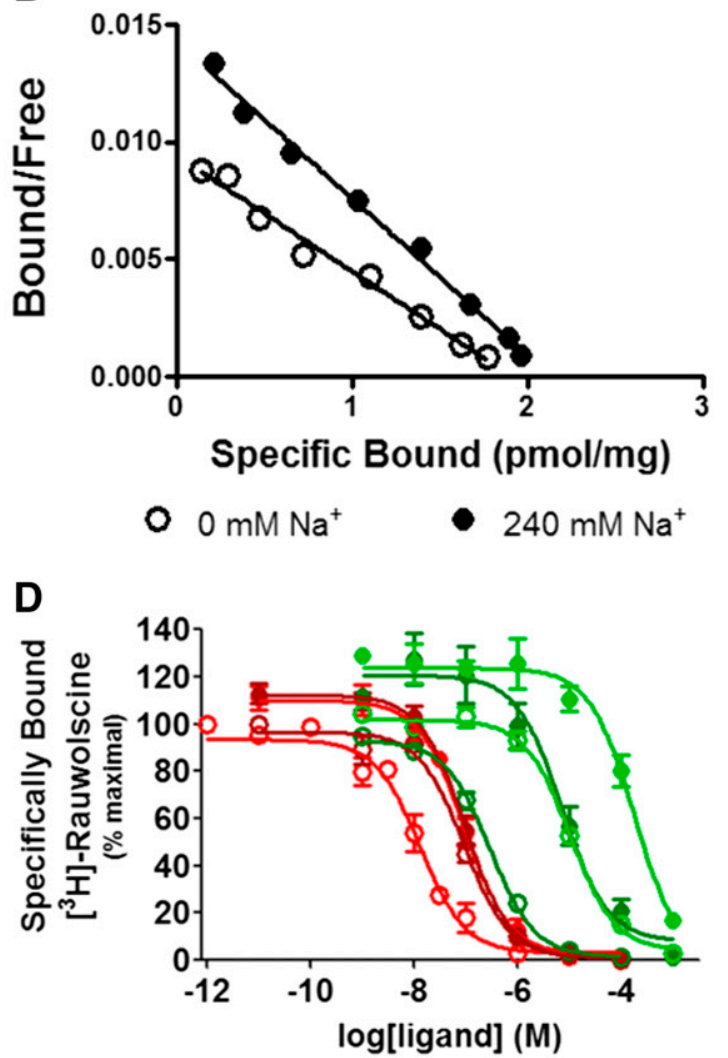

- Dexmedetomidine - Levomedetomidine

$-(-)-N E$

- $0 \mathrm{mM} \mathrm{Na}^{+}$

Fig. 6. Sodium increases the affinity of $\left[{ }^{3} \mathrm{H}\right]$ rauwolscine for the BiOctR. Saturation isotherms were performed using HEK293 cells expressing either BiOctR or human $\alpha_{2 \mathrm{C}}-\mathrm{AR}$ as the source of the receptors and $\left[{ }^{3} \mathrm{H}\right]$ rauwolscine as the radioligand. Binding buffer for the BiOctR contained $50 \mathrm{mM}$ Tris, $\mathrm{pH}$ 7.4, supplemented with either 15 or $240 \mathrm{mM}$ sodium, whereas, for the $\alpha_{2 \mathrm{C}}-\mathrm{AR}$, the binding buffer was $50 \mathrm{mM}$, Tris $\mathrm{pH}$, in either the absence or presence of $240 \mathrm{mM}$ sodium. The data were transformed and graphed as Scatchard plots. A Student's $t$ test was performed to assess statistical significance between high and low sodium conditions. (A) Representative example of a Scatchard plot of $\left[{ }^{3} \mathrm{H}\right]$ rauwolscine saturation isotherm binding in the presence of 15 or $240 \mathrm{mM}$ sodium at the BiOctR. $\mathrm{B}_{\max }$ and $\mathrm{K}_{\mathrm{D}}$ in the presence of $15 \mathrm{mM}$ sodium were $2.1 \pm 0.4 \mathrm{pmol} / \mathrm{mg}$ and $12.3 \pm 1.2 \mathrm{nM}$, respectively. $\mathrm{B}_{\max }$ and $\mathrm{K}_{\mathrm{D}}$ in the presence of $240 \mathrm{mM} \mathrm{Na}^{+}$were $2.3 \pm 0.5 \mathrm{pmol} / \mathrm{mg}$ and $3.4 \pm 0.3 \mathrm{nM}$, respectively. The measured $\mathrm{B}_{\max }$ values were not significantly different $(P>0.05)$ between the two conditions, but the $\mathrm{K}_{\mathrm{D}}$ values were $(P<0.05)$. The dashed lines represent extrapolation of the linear best fit lines to the $x$-axis intercept. (B) Representative example of Scatchard plots of $\left[{ }^{3} \mathrm{H}\right]$-rauwolscine in the presence of 0 or $240 \mathrm{mM} \mathrm{Na}{ }^{+}$at the $\alpha_{2 \mathrm{C}}-\mathrm{AR}$. $\mathrm{B}_{\max }$ and $\mathrm{K}_{\mathrm{D}}$ in the presence of $0 \mathrm{mM}$ $\mathrm{Na}^{+}$were $2.5 \pm 0.5 \mathrm{pmol} / \mathrm{mg}$ and $0.23 \pm 0.03 \mathrm{nM}$, respectively. $\mathrm{B}_{\max }$ and $\mathrm{K}_{\mathrm{D}}$ in the presence of $240 \mathrm{mM} \mathrm{Na} \mathrm{mere}^{+} .0 \pm 0.4 \mathrm{pmol} / \mathrm{mg}$ and $0.17 \pm 0.02 \mathrm{nM}$, respectively. Neither $B_{\max }$ nor $K_{D}$ values were significantly different between the two binding conditions $(P>0.05)$. (C) Inhibition curves for dexmedetomidine, levomedetomidine, and octopamine in the presence of low $(15 \mathrm{mM})$ or high $(240 \mathrm{mM}) \mathrm{Na}^{+}$at the BiOctR. The $\mathrm{K}_{\mathrm{i}}$ values in the presence of low and high $\mathrm{Na}^{+}$are as follows: dexmedetomidine $=11 \pm 6.2$ versus $10 \pm 1.9 \mathrm{nM}$; levomedetomidine $=367 \pm 45.2$ versus $135 \pm 48.6 \mathrm{nM}$; octopamine $=$ $26,380 \pm 8120$ versus 40,820 $\pm 20,610 \mathrm{nM}$. (D) Inhibition curves for dexmedetomidine, levomedetomidine, ( - )-NE, and (+)-NE in the absence or presence of $\mathrm{Na}^{+}$at the $\alpha_{2 \mathrm{C}}$ receptor. The $\mathrm{K}_{\mathrm{i}}$ values in the absence or presence of $\mathrm{Na}^{+}$are as follows: dexmedetomidine $=5.5 \pm 1.5$ versus $36.9 \pm 8.1 \mathrm{nM}$; levomedetomidine $=26.8 \pm 4.4$ versus $28.0 \pm 4.0 ;(-)-\mathrm{NE}=116.2 \pm 15.7$ versus $3770 \pm 1255 \mathrm{nM} ;(+)-\mathrm{NE}=4106 \pm 670$ versus $727,000 \pm 28,878 \mathrm{nM}$. Averaged data for all experiments for groups are plotted as the mean \pm S.E.M. from two or more independent experiments. 

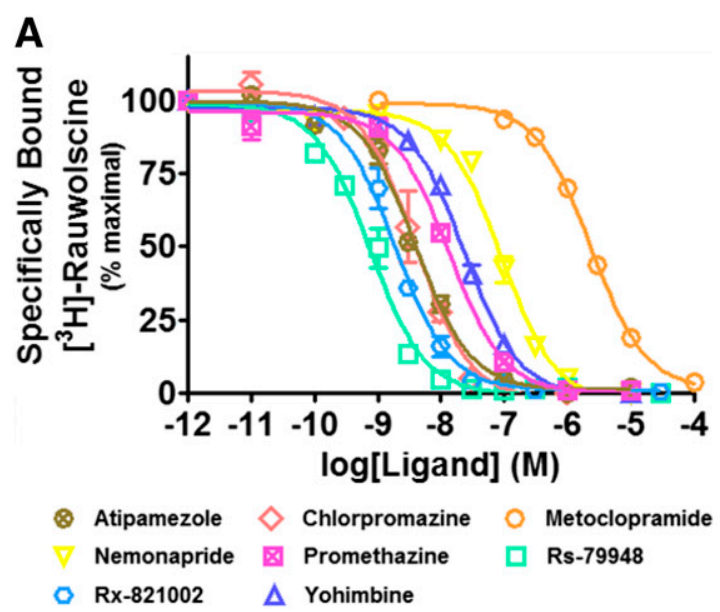

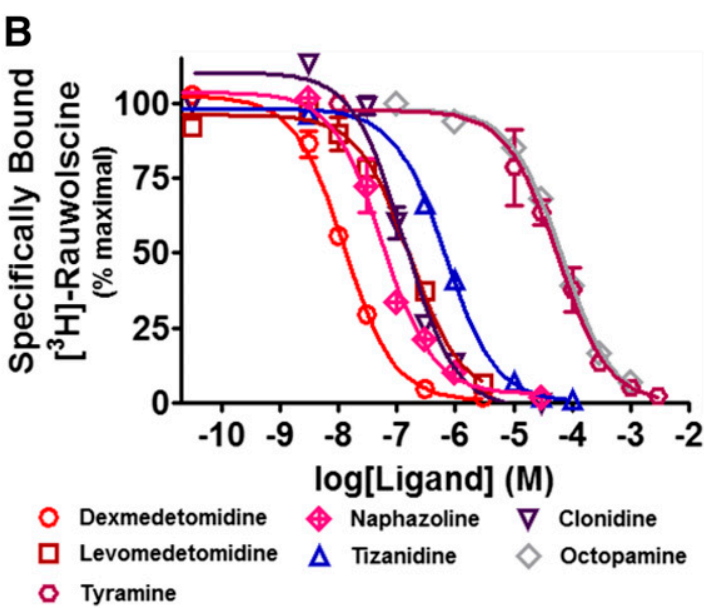

Fig. 7. Mammalian GPCR antagonists have higher affinity than agonists at the BiOctR in the presence of high salt concentration. Competition-type radioligandbinding assays were performed to measure the affinities of the ligands. HEK293 cells expressing the BiOctR served as the source of the membranes, and $\left[{ }^{3} \mathrm{H}\right]$ rauwolscine was used as the radioligand. Assay was performed in the presence of $240 \mathrm{mM} \mathrm{Na}$. The $\mathrm{K}_{\mathrm{i}}$ was calculated from the $\mathrm{IC}_{50}$ using the Cheng-Prusoff equation. (A and B) Inhibition curves for antagonists and agonists, respectively. Averaged data for all experiments for all groups are plotted as the mean \pm S.E.M. from two or more independent experiments. The $\mathrm{K}_{\mathrm{i}}$ values are listed in Table 2. See Supplemental Table 1 for chemical structures.

tested was similar between both the BiOctR and DmOctR with the antagonists chlorpromazine, promethazine, rauwolscine, Rs-79948, Rx-821002, and yohimbine completely and nemonapride and metoclopramide only partially reversing the response induced by $1 \mu \mathrm{M}$ octopamine at the solubility limits at which they could be tested in this assay system.

Ligands that functioned as agonists were evaluated further by generating concentration-response curves to estimate their potencies at the OctRs (Fig. 2, E and F; see Table 1 for $\mathrm{EC}_{50}$ values). The rank order potencies $\left(\mathrm{EC}_{50}\right.$ values) at BiOctR were dexmedetomidine $>>$ naphazoline $\approx$ tizanidine $\approx$ clonidine $>$ levomedetomidine $>$ octopamine $>$ tyramine $\approx$ $(-)$-EPI $>(-)$-NE, whereas the rank order potencies at the DmOctR were dexmedetomidine $>$ naphazoline $>$ clonidine $>$ tizanidine $\approx$ octopamine $>(-)$-EPI $>(-)$-NE. Although rank order agonist potencies were similar between the two receptors, large differences were noted for levomedetomidine and tyramine, whereas at DmOctR, levomedetomidine had no significant activity at the highest concentration that could be tested $(10 \mu \mathrm{M})$ and tyramine was only able to induce a $12 \%$ response at a maximum concentration of $100 \mu \mathrm{M}$ (Fig. 2B). Major differences in agonist efficacies between these species were noted as well (see Table 1 for efficacies). At the BiOctR, levomedetomidine, (-)-EPI, (-)-NE, and tyramine were partial agonists, and the rest were full agonists. In contrast, at DmOctR the imidazolines behaved as partial agonists, and (-)-EPI and (-)-NE had significantly higher efficacies than octopamine, with (-)-EPI, in particular, acting as a superagonist. When the potencies at the BiOctR and DmOctR are plotted against one another, two clusters can be seen: one for the less potent biogenic amines and one for the more potent imidazoles/imidazolines (Fig. 3A). With the exception of tyramine and levomedetomidine, for which potencies could not be measured at DmOctR, there is a linear relationship between the two receptors with a Pearson's $r^{2}$ of 0.91 and a slope of $0.92 \pm 0.13$, further demonstrating that the two receptors share a similar pharmacological profile. In contrast, the agonist efficacy profile had a poor correlation (Pearson's $r^{2}=0.63$ ), consistent with dramatic differences in the efficacy profiles (Fig. 3B).

OctR Agonists Induce Hyperactivity in Barnacle Cyprids. The six most potent synthetic agonists of the
BiOctR were additionally tested for their ability to induce hyperactivity responses in barnacle cyprids (Fig. 4). Dexmedetomidine was able to induce hyperactivity in a concentration-dependent manner, and the response to $1 \mu \mathrm{M}$ dexmedetomidine was statistically significant compared with vehicle $(P<0.05)$ and was blocked by coapplication of the OctR antagonist atipamezole $(30 \mu \mathrm{M})$ (Fig. 4A). When tested alone at the same concentration $(30 \mu \mathrm{M})$, atipamezole's effect was no different than the vehicle control (Fig. 4B). Treatment with levomedetomidine resulted in an increase in the number of abrupt cyprid body movements, but the response did not reach statistical significance most likely due to its lower rank order potency. Clonidine, naphazoline, and tizanidine induced significant hyperactivity responses in cyprids that could be reversed by atipamezole, but an equimolar comparison suggests they were not as effective as dexmedetomidine. The endogenous agonist, octopamine, also induced a hyperactivity response in cyprids

TABLE 2

Affinities of mammalian GPCR antagonists and agonists at the BiOctR $\left[{ }^{3} \mathrm{H}\right]$ Rauwolscine in the presence of $240 \mathrm{mM} \mathrm{Na}^{+}$was used to perform competition curves for the different ligands. The $\mathrm{K}_{\mathrm{i}}$ values were calculated from the $\mathrm{IC}_{50}$ values using the Cheng-Prusoff equation. Values represent mean \pm S.E.M.

\begin{tabular}{lc}
\hline \multicolumn{1}{c}{ Ligand } & $\begin{array}{c}\text { Affinity, } \mathrm{K}_{\mathrm{i}} \\
\text { (nM } \pm \text { S.E.M.) }\end{array}$ \\
\hline Antagonists & \\
Rs-79948 & $0.59 \pm 0.076$ \\
Rx-821002 & $1.5 \pm 0.15$ \\
Chlorpromazine & $2.9 \pm 0.56$ \\
Atipamezole & $3.0 \pm 0.38$ \\
Rauwolscine & $4.6 \pm 0.50$ \\
Promethazine & 10.1 .4 \\
Yohimbine & $17 \pm 1.5$ \\
Nemonapride & $66 \pm 10$. \\
Metoclopramide & $1700 \pm 71$ \\
Agonists & \\
Dexmedetomidine & $8.2 \pm 0.75$ \\
Naphazoline & $42 \pm 7.8$ \\
Clonidine & $99 \pm 22$ \\
Levomedetomidine & $101 \pm 19$ \\
Tizanidine & $580 \pm 40$ \\
Octopamine & $43,000 \pm 3300$ \\
Tyramine & $45,000 \pm 12,000$ \\
\hline
\end{tabular}



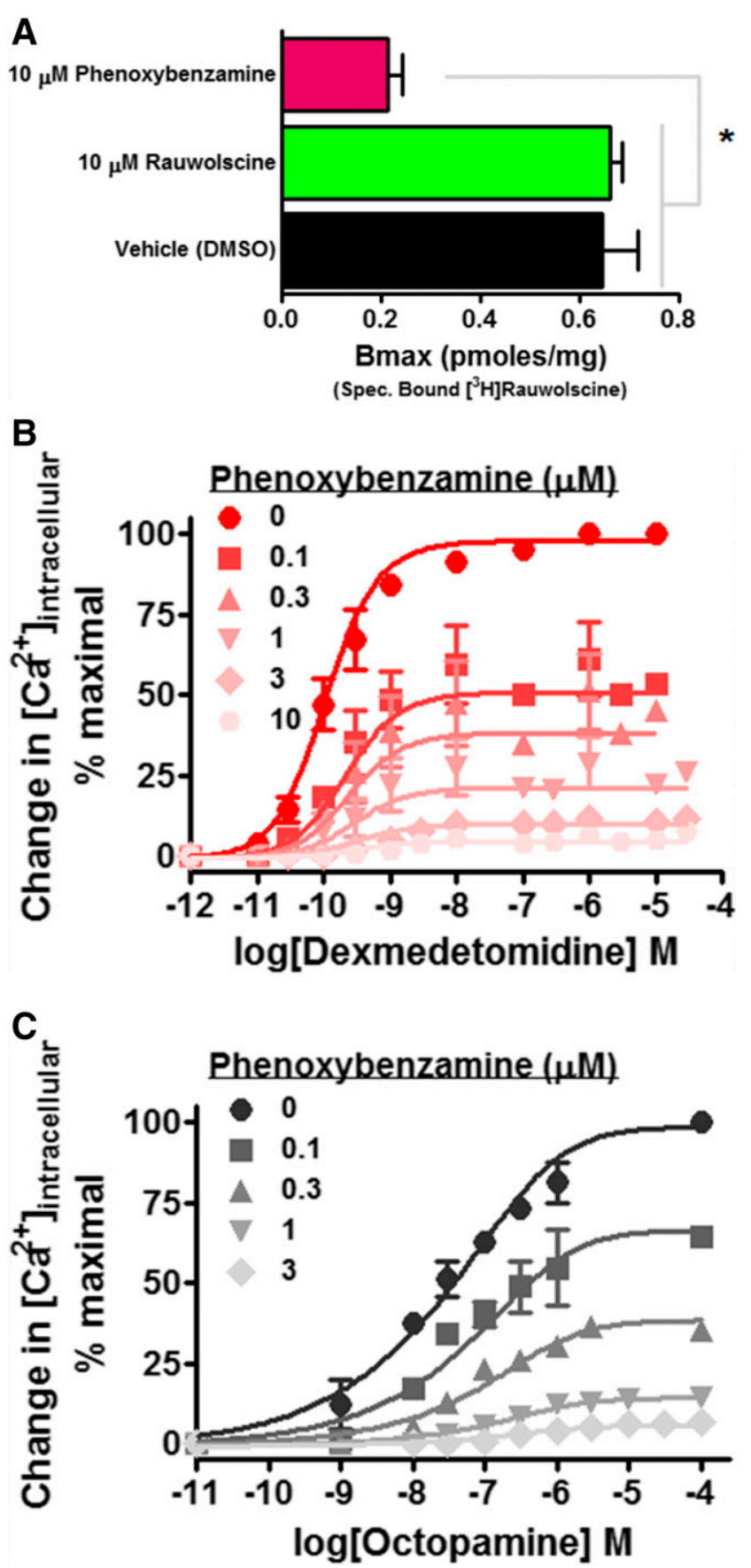

Fig. 8. Phenoxybenzamine is an irreversible antagonist, and BiOctR receptor reserve has a modest effect on agonist potency. (A) BiOctRexpressing cells were exposed to either vehicle, $10 \mu \mathrm{M}$ rauwolscine, or $10 \mu \mathrm{M}$ phenoxybenzamine for $\sim 12$ hours. The treated cells were then thoroughly washed, and then radioligand binding was performed using $\left[{ }^{3} \mathrm{H}\right]$ rauwolscine. Rauwolscine pretreatment had no effect on the $\mathrm{B}_{\max }$, but phenoxybenzamine lowered the $\mathrm{B}_{\max }$ by $\sim 3$-fold ( ${ }^{*} P<0.05$ compared to vehicle (DMSO) and $10 \mu \mathrm{M}$ rauwolscine), indicating that it irreversibly interacts with the BiOctR. See Supplemental Table 7 numerical values for the bar graphs shown in (A-C). The affinity of the agonists was measured by applying the operational model of agonism. The BiOctR $\mathrm{B}_{\max }$ was depleted using the irreversible antagonist phenoxybenzamine, followed by stimulation with increasing concentrations of the agonists to generate concentration-response curves for each concentration of phenoxybenzamine. The calculated agonist potencies $\left(\mathrm{K}_{\mathrm{A}}\right)$ were $(B) 0.3 \pm 0.1 \mathrm{nM}$ for dexmedetomidine and (C) $588 \pm 226 \mathrm{nM}$ for octopamine. Averaged data for all experiments for all groups are plotted as the mean \pm S.E.M. from two to three independent experiments. that could be blocked by atipamezole; however, high micromolar concentrations were required.

BiOctR Exhibits Sodium Sensitivity for Both Agonists and Antagonists. Previous binding studies on OctRs from cockroach, fly, mosquito, and snail employed either $\left[{ }^{3} \mathrm{H}\right]$ yohimbine or $\left[{ }^{3} \mathrm{H}\right]$ rauwolscine as the radioligands and used $50 \mathrm{mM}$ Tris, $\mathrm{pH}$ 7.4, as the binding buffer (Gerhardt et al., 1997; Bischof and Enan, 2004; Enan, 2005; Kastner et al., 2014). When the same binding condition was used for the BiOctR, no specific binding could be measured (Fig. 5). Addition of $480 \mathrm{mM} \mathrm{Na}{ }^{+}$to the binding buffer to mimic the approximate sodium concentration in marine hemolymph (Castille and Lawrence, 1981) resulted in a large increase in $\left[{ }^{3} \mathrm{H}\right]$ rauwolscine-specific binding, which was comparable to the specific binding obtained when 35 ppt marine salt was used as the binding buffer (Fig. 5A). The additional inclusion either $\mathrm{K}^{+}$or $\mathrm{K}^{+}$and $\mathrm{Mg}^{2+}$ to the buffer containing $480 \mathrm{mM} \mathrm{Na}^{+}$ did not enhance the binding of the radioligand further (Fig. 5A). Next, specific binding as a function of sodium concentration was measured to identify an optimal sodium concentration. The lowest $\mathrm{Na}^{+}$concentration that produced a significant measurable $\left[{ }^{3} \mathrm{H}\right]$ rauwolscine-specific binding was $15 \mathrm{mM}$, and then there was a concentration-dependent increase in the specific binding that started to plateau at about $240 \mathrm{mM}$ (Fig. $5 \mathrm{~B})$. For this reason, all subsequent binding experiments were performed in the presence of the lowest optimal concentration of $240 \mathrm{mM} \mathrm{Na}{ }^{+}$. In contrast, no specific binding for $\left[{ }^{3} \mathrm{H}\right]$ rauwolscine at the DmOctR could be measured either in the absence or presence of sodium, and, consequently, no further radioligand-binding studies were performed with the DmOctR.

To determine the accessibility of the sodium site, binding assays were next performed using whole cells, and the mammalian physiologic buffer HBSS (Fig. 5C), as previous experiments had been performed on broken membrane preparations. Two variants of the buffer were used: one containing $140 \mathrm{mM} \mathrm{NaCl}$, and the other containing $140 \mathrm{mM}$ of the sodium substitute NMDG to maintain charge and osmolarity gradients. The $\left[{ }^{3} \mathrm{H}\right]$ rauwolscine-specific binding in the whole-cell configuration under either buffer condition was not significantly different $(P>0.05$, Student's $t$ test), indicating the importance of intracellular sodium concentration. To rule out the possibility that sodium's effect on binding might be influenced by charge, $\left[{ }^{3} \mathrm{H}\right]$ rauwolscine binding was conducted on broken membrane preparations in the presence of either $240 \mathrm{mM} \mathrm{Na}^{+}$or $240 \mathrm{mM} \mathrm{NMDG}$ (Fig. 5D). Similar to the binding in the absence of sodium, no $\left[{ }^{3} \mathrm{H}\right]$ rauwolscine-specific binding could be measured in the presence of $240 \mathrm{mM}$ NMDG. A potassium phosphate buffer was also tested, as binding under these conditions had been reported in a previous study using BiOctR heterologously expressed in mammalian cells and $\left[{ }^{3} \mathrm{H}\right]$-Rs79948-197 as the radioligand (Lind et al., 2010); however, no $\left[{ }^{3} \mathrm{H}\right]$ rauwolscine-specific binding was observed using this buffer. To determine whether sodium was influencing the affinity $\left(\mathrm{K}_{\mathrm{D}}\right)$ of $\left[{ }^{3} \mathrm{H}\right]$ rauwolscine, the apparent $\mathrm{B}_{\max }$, or both, saturation isotherms were performed in the presence of low $(15 \mathrm{mM})$ or high $(240 \mathrm{mM})$ sodium. Sodium had no significant effect on $B_{\max }$, but decreased the $K_{D}$ value for $\left[{ }^{3} \mathrm{H}\right]$ rauwolscine by more than 3 -fold, indicating an increased affinity for the receptor (Fig. 6A). For comparison, the sodium sensitivity of $\left[{ }^{3} \mathrm{H}\right]$ rauwolscine-specific binding to the human $\alpha_{2 \mathrm{C}}-\mathrm{AR}$ was tested because rauwolscine is a high-affinity 
$\alpha$-adrenergic receptor antagonist. In contrast to what was observed at the BiOctR, sodium did not significantly alter the $\mathrm{B}_{\text {max }}$ or $\mathrm{K}_{\mathrm{D}}$ of $\left[{ }^{3} \mathrm{H}\right]$ rauwolscine at a cloned vertebrate $\alpha_{2 \mathrm{C}}-\mathrm{AR}$ (Fig. 6B). Because reductions in agonist affinity in the presence of $\mathrm{Na}^{+}$have been reported previously for some mammalian biogenic amine GPCRs (Katritch et al., 2014), the effect of sodium on agonist affinity at the OctRs was investigated. Because at the BiOctR measurable $\left[{ }^{3} \mathrm{H}\right]$ rauwolscine binding could not be detected in the absence of $\mathrm{Na}^{+}$, agonist inhibition curves were performed in the presence of either the lowest concentration of sodium that yielded measurable specific $\left[{ }^{3} \mathrm{H}\right]$ rauwolscine binding $(15 \mathrm{mM})$ or the lowest concentration of sodium that yielded optimal specific $\left[{ }^{3} \mathrm{H}\right]$ rauwolscine binding (240 mM). Under these two conditions, sodium had no effect on the affinities of dexmedetomidine, levomedetomidine, or octopamine (Fig. 6C). In contrast, at the $\alpha_{2 \mathrm{C}}$ receptor whose binding to $\left[{ }^{3} \mathrm{H}\right]$ rauwolscine is sodium-insensitive, the affinities of dexmedetomidine, (-)-NE, and (+)-NE decreased by more than 6-fold in the presence of sodium, but had no effect on levomedetomidine (Fig. 6D).

Affinities $\left(\mathrm{K}_{\mathrm{i}}\right)$ of agonists and antagonist at the BiOctR were measured by a $\left[{ }^{3} \mathrm{H}\right]$ rauwolscine competition-type radioligandbinding assay (Fig. 7; see Table 2 for affinities). Affinities for the antagonists ranged from 0.6 to $1700 \mathrm{nM}$, and the rank order was as follows: Rs-79948 > Rx-821002 > chlorpromazine $\approx$ atipamezole $\approx$ rauwolscine $>$ promethazine $\approx$ yohimbine $>$ nemonapride $>$ metoclopramide. Affinities for the agonists ranged from 8.2 to $44,840 \mathrm{nM}$ with an agonist rank order of dexmedetomidine $>$ naphazoline $>$ clonidine $>$ tizanidine $>$ octopamine $\approx$ tyramine. When the affinities $\left(\mathrm{K}_{\mathrm{i}}\right)$ of the agonists are compared with their potencies, the potencies were 12 -fold to 1500 -fold higher. Due to the large discrepancies between the potencies and affinities, the possibility that receptor reserve could account for the differences was tested using the operational model of agonism. First employing washout experiments, it was demonstrated that phenoxybenzamine functioned an irreversible ligand at the BiOctR, whereas rauwolscine was a reversible ligand (Fig. $8 \mathrm{~A})$. Because the operational model of agonist requires depletion of receptor density, phenoxybenzamine was used to decrease the receptor density, and functional concentration responses were generated for dexmedetomidine and octopamine (Fig. 8, $\mathrm{B}$ and $\mathrm{C}$, respectively). In theory, as $\mathrm{B}_{\max }$ approaches zero, the potency equals the agonist affinity. The measured agonist affinity values for dexmedetomidine and octopamine were 24-fold and 74-fold higher than their $\mathrm{K}_{\mathrm{i}}$ values, respectively, and the measured potencies for dexmedetomidine and octopamine were 3-fold and 21-fold higher than their $K_{A}$ values. Because of the large difference between the $K_{A}$ and $K_{i}$ values, we compared the antagonist affinity for rauwolscine measured using radioligand binding $\left(\mathrm{K}_{\mathrm{i}}\right)$ with the antagonist dissociation constant $\mathrm{K}_{\mathrm{B}}$ measured using a functional approach (Fig. 9, B and C). As the concentration of rauwolscine was increased, there was a progressive rightward shift in the potency of dexmedetomidine, as well as a decrease in efficacy at both the BiOctR and DmOctR. To obtain a $K_{B}$ value from this experiment, the curves have to be transformed into a Schild plot. However, this model requires that the efficacy remain constant, and, because this requirement was violated, the $K_{B}$ for rauwolscine obtained from such an analysis would be faulty. Similarly, at the $\alpha_{2 \mathrm{C}}-\mathrm{AR},\left[{ }^{3} \mathrm{H}\right]$ rauwolscine saturation isotherms in the presence of increasing concentrations of dexmedetomidine showed that as the concentration of dexmedetomidine increased, there was an increase in $K_{D}$ (decrease in $\left[{ }^{3} \mathrm{H}\right]$ rauwolscine affinity) and a decrease in $\mathrm{B}_{\max }$ (Fig. 9A), yet a statistically significant change in these parameters was only observed in the absence and the presence of $100 \mathrm{nM}$ dexmedetomidine $(P<0.05$, one-way analysis of variance, Bonferroni's post hoc).

\section{Discussion}

A number of new findings are reported in this study. First, the radioligand-binding experiments clearly demonstrated that the BiOctR exhibits sodium sensitivity for $\left[{ }^{3} \mathrm{H}\right]$ rauwolscine, in which sodium increases the receptor's affinity for this antagonist while having no effect on apparent $B_{\max }$. Second, the receptor reserve only had a comparatively small effect on agonist potency, which suggests that the receptor also exhibits sodium sensitivity for agonists, but, in contrast to rauwolscine, sodium decreases the affinity for agonists. Third, rauwolscine binds to a different binding site than the agonist dexmedetomidine. It was also shown that both the BiOctR and the DmOctR have a preference for the $(+)$-enantiomer of medetomidine and the (-)-enantiomer of $\mathrm{NE}$, so much so in the case of medetomidine that no significant interactions of levomedetomidine could be detected at the DmOctR. Furthermore, all of the imidazolines tested in this study functioned as full agonists, and (-)-EPI and (-)-NE functioned as strong partial agonists at the BiOctR, whereas, at the DmOctR, the imidazolines clonidine, naphazoline, and tizanidine were partial agonists and (-)-EPI and (-)-NE functioned as low-potency superagonists. We also show that all of the imidazolines tested, as well as dexmedetomidine and octopamine, induce hyperactivity in barnacle cyprids, providing further support for the hypothesis that OctR agonists in general are likely to have deterrent properties, making them useful for preventing biofouling by barnacles.

TABLE 3

Residues that line the presumed orthosteric (agonist) site of the BiOctR and DmOctR differ from the human $\alpha_{2 \mathrm{C}}$ receptor by five amino acids

The protein sequences of the three receptors were aligned using CLUSTALW, and the active site residues of the $\alpha_{2 \mathrm{C}}-\mathrm{AR}$ were compared with those of the BiOctR and DmOctR. Numbers in brackets are according to the Ballesteros-Weinstein numbering convention (Ballesteros and Weinstein, 1995).

\begin{tabular}{|c|c|c|c|}
\hline $\begin{array}{c}\text { Amino Acid Number in the } \\
\alpha_{2 \mathrm{C}} \mathrm{AR} \text { and Relative Position }\end{array}$ & $\alpha_{2 \mathrm{C}}-\mathrm{AR}$ & BiOctR & DmOctR \\
\hline 134 [3.35] & Phe (F) & Met (M) & Met $(\mathrm{M})$ \\
\hline $178[4.52]$ & Ile (I) & Leu (L) & Leu (L) \\
\hline 202 [5.30] & Cys (C) & $\mathrm{Gln}(\mathrm{E})$ & $\mathrm{Gln}(\mathrm{E})$ \\
\hline 215 [5.43] & Cys (C) & Ala (A) & Ala (A) \\
\hline $422[7.38]$ & Phe $(\mathrm{F})$ & Phe $(\mathrm{F})$ & Leu (L) \\
\hline
\end{tabular}




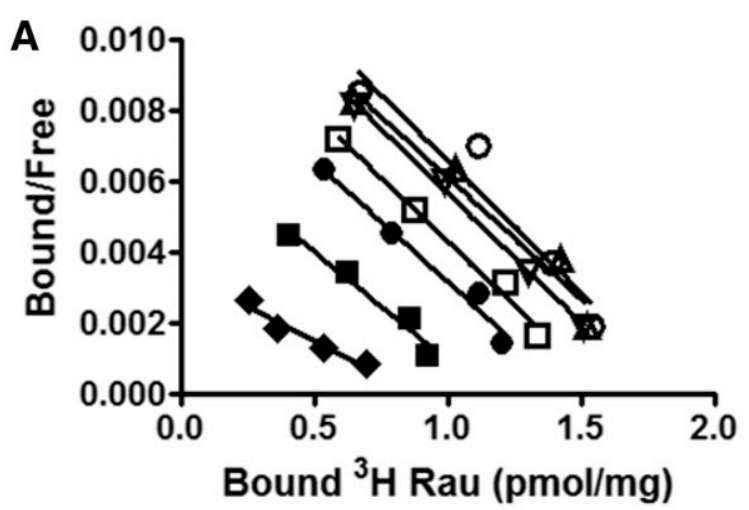

- $0 \mathrm{nM}$ Dexmedetomidine

$\nabla 1 \mathrm{nM}$ Dexmedetomidine

$\Delta \quad 0.3 \mathrm{nM}$ Dexmedetomidine

- $10 \mathrm{nM}$ Dexmedetomidine

$3 \mathrm{nM}$ Dexmedetomidine

$100 \mathrm{nM}$ Dexmedetomidine

B

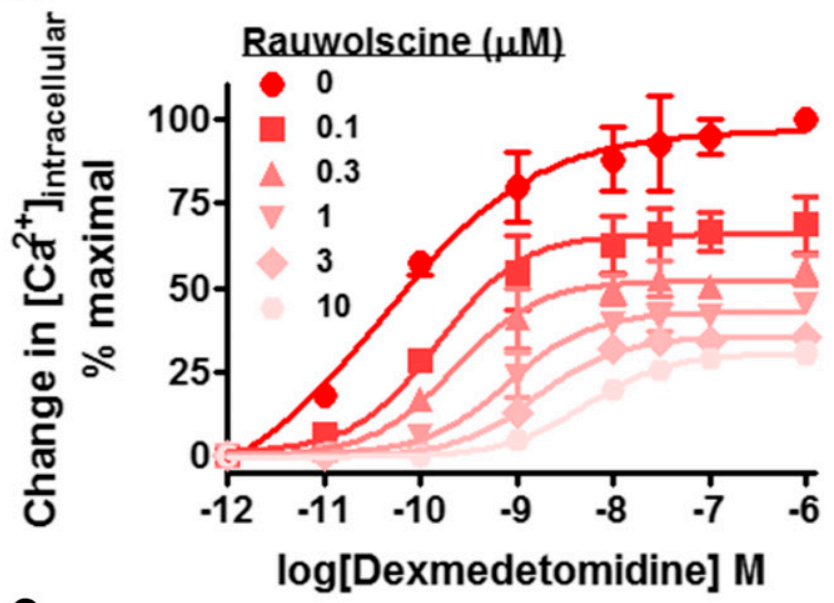

C

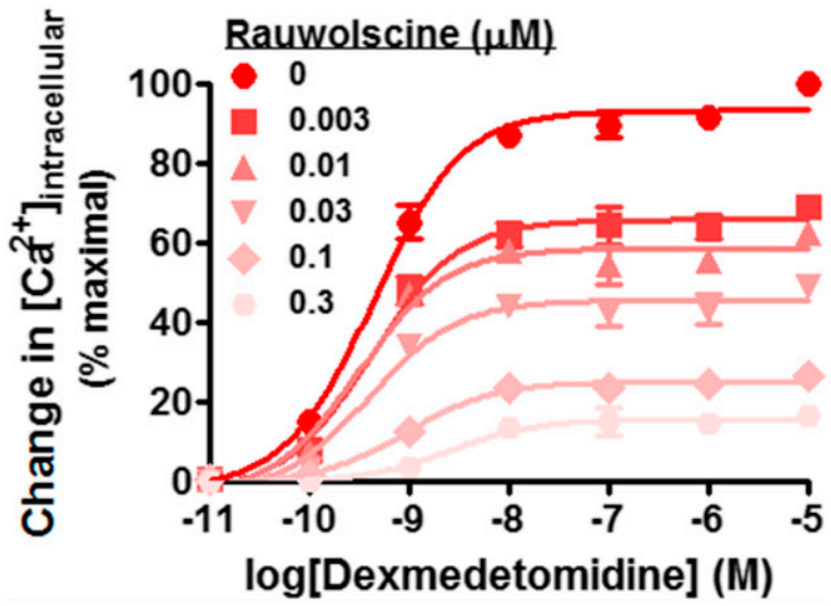

Fig. 9. Rauwolscine interacts with the $\alpha_{2 \mathrm{C}} \mathrm{AR}, \mathrm{BiOctR}$, and DmOctR at an allosteric site. To evaluate whether rauwolscine and dexmedetomidine are competing for the same site on the $\alpha_{2 \mathrm{C}}-\mathrm{AR},\left[{ }^{3} \mathrm{H}\right]$ rauwolscine saturation isotherms were performed in the presence of increasing concentration of dexmedetomidine. To evaluate whether rauwolscine and dexmedetomidine are competing for the same site on the BiOctR or the DmOctR, the functional antagonism of rauwolscine for dexmedetomidine was evaluated. HEK293 cells expressing either the BiOctR or the DmOctR were pretreated with various fixed concentrations of the antagonist rauwolscine and then stimulated with the agonist dexmedetomidine over its entire concentration range. (A) At the $\alpha_{2 \mathrm{C}}-\mathrm{AR}, 100 \mathrm{nM}$ dexmedetomidine
Although the antagonist profile as assessed by functional assays was essentially the same for both BiOctR and the DmOctR, there were some notable differences in the agonist profiles. Reasons for these differences are unknown, but likely possibilities include differences in key residues involved in signal transduction with $\mathrm{G}_{\mathrm{q}}$ proteins, or differences in the amino acids presumed to line the binding site crevice, resulting in ligands adopting a different orientation in the binding pocket. Of the 26 known amino acids that line the presumed $\alpha_{2 \mathrm{C}} \mathrm{AR}$ active site (Kumar et al., 2014), the BiOctR and DmOctR protein sequences differ at only five positions, and, in one of these positions (i.e., 4.52), the differences are due to a conserved amino acid substitution (Table 3 ). These differences could also account for the other differences discovered in our radioligand-binding studies.

Our radioligand-binding studies demonstrated that the BiOctR exhibits sodium sensitivity and that this site is intracellularly accessible, which is consistent with what has been previously demonstrated for mammalian sodiumsensitive GPCRs (Neve, 1991; Neve et al., 2001; Schetz and Sibley, 2001). If the sodium site were accessible from the extracellular side, then the receptor would chronically be in an agonist low-affinity state and would require an exceedingly high concentration of the agonist to elicit any response. From a physiologic and molecular mechanistic perspective then, it makes intuitive sense that the sodium site would be accessible only from the intracellular side, and provides a feedback mechanism for coupling electrical excitability with chemical excitability. Specifically, depolarization of the cell following a sodium-dependent action potential, for example, might increase the local concentration of intracellular sodium near the internal surface of the membrane enough to switch any sodium-sensitive receptor to the agonist low-affinity state, effectively encouraging agonist dissociation and attenuation of the agonist response (Schetz, 2005).

In the only other report investigating BiOctR, a $50 \mathrm{mM}$ potassium phosphate buffer ( $\mathrm{pH}$ 7.4) was used as the radioligand-binding buffer with $\left[{ }^{3} \mathrm{H}\right]-\mathrm{Rs} 79948-197$ as the radioligand (Lind et al., 2010). In that report, the affinity $\left(\mathrm{K}_{\mathrm{D}}\right)$ of $\left[{ }^{3} \mathrm{H}\right]-\mathrm{Rs} 79948$ was measured to be $7.3 \mathrm{nM}$; however, in this study, the affinity $\left(\mathrm{K}_{\mathrm{i}}\right)$ of $\mathrm{Rs} 79948$ was measured to be $0.6 \pm 0.08 \mathrm{nM}$. When we tested $\left[{ }^{3} \mathrm{H}\right]$ rauwolscine using the same phosphate buffer, we were unable to detect measurable binding. Because we demonstrated that the affinity of rauwolscine increases in the presence of sodium, it is likely that we measured approximately a 12-fold higher affinity for Rs79948 than previously reported due to the presence of sodium, and that Lind et al. (2010) were able to measure

increased the $\mathrm{K}_{\mathrm{D}}$ (i.e., decreased the affinity) and decreased the $\mathrm{B}_{\max }$ for $\left[{ }^{3} \mathrm{H}\right]$ rauwolscine $(P<0.05$, one-way analysis of variance, followed by Bonferroni's post hoc). A representative example is shown in (A). The respective averaged $\mathrm{K}_{\mathrm{D}}\left(\mathrm{pM} \pm\right.$ S.E.M.) and $\mathrm{B}_{\max }(\mathrm{pmol} / \mathrm{mg} \pm$ S.E.M.) values calculated from three independent experiments in the absence and presence of $0.3,1,3,10,30$, and $100 \mathrm{nM}$ dexmedetomidine, respectively, are $K_{\mathrm{D}}=130 \pm 0.20,160 \pm 12,150 \pm 12,180 \pm 20,200 \pm 29,220 \pm 31$, and $270 \pm 18$, and $\mathrm{B}_{\max }=2.1 \pm 0.21,2.0 \pm 0.073,2.1 \pm 0.16,2.0 \pm 0.26,1.8 \pm$ $0.19,1.4 \pm 0.18$, and $1.0 \pm 0.16$. Rauwolscine progressively decreased both the efficacy and potency of dexmedetomidine at both (B) BiOctR and (C) DmOctR, consistent with rauwolscine exerting a noncompetition interaction at an allosteric site. Averaged data are plotted as the mean \pm S.E.M. from two to three independent experiments. 
binding in the absence of sodium because, under this binding condition, $\left[{ }^{3} \mathrm{H}\right]-\mathrm{Rs} 79948-197$ has a high enough affinity for BiOctR to be detected in a rapid filtration radioligand-binding assay, whereas $\left[{ }^{3} \mathrm{H}\right]$ rauwolscine does not. This implies that, like rauwolscine, the affinity for BiOctR of the structurally similar Rs79948 is also likely to be sodium-sensitive.

Sodium is a well-known allosteric modulator of GPCRs (Blume et al., 1979; Limbird et al., 1982), and, although it is unusual for antagonists to display marked sodium sensitivity, it is not unprecedented (Neve, 1991; Malmberg et al., 1993; Schetz et al., 1999). The sodium sensitivity of $\left[{ }^{3} \mathrm{H}\right]$ rauwolscine appears to be unique to the BiOctR because sodium had no significant effect on the $\mathrm{K}_{\mathrm{D}}$ or $\mathrm{B}_{\max }$ of rauwolscine at its evolutionary homolog, the $\alpha_{2 \mathrm{C}}$-AR. In the case of agonists, previous studies have shown that sodium decreases agonist affinity for GPCRs (Limbird et al., 1982; Selley et al., 2000) by putting the receptor in an agonist low-affinity state. As expected, at the $\alpha_{2 \mathrm{C}}-\mathrm{AR}$, sodium decreased the affinity of dexmedetomidine, (-)-NE, and (+)-NE; however, sodium had no effect on the affinity of levomedetomidine, suggesting that these antipodes truly have a difference in sodium sensitivity. By analogy with our previous work on mammalian $\mathrm{D}_{2}$ dopamine receptors and conformational changes induced by sodium (Ericksen et al., 2009), the difference in sodium sensitivity for dexmedetomidine versus levomedetomidine at the $\alpha_{2}$-AR suggests these antipodes either assume a different orientation in the binding pocket, at least with respect to the top of the TM2/TM3 interface, or that the $\beta$-hydroxyl group of dexmedetomidine points toward and that of levomedetomidine away from the TM2/TM3 interface.

In contrast to the $\alpha_{2 \mathrm{C}}-\mathrm{AR}$, no sodium sensitivity was apparent for agonist affinities at the BiOctR, and, furthermore, the agonist potencies were 12-fold to more than 1000-fold higher than the corresponding affinities. By utilizing the operational model of agonism (Leff et al., 1985; Black et al., 2010), it was demonstrated that receptor reserve (substantial amounts of excess receptor) had a small effect on dexmedetomidine ( $\sim 3$-fold) potency, but a larger effect on octopamine ( $\sim 20$-fold) potency. Furthermore, when the affinities calculated by the operational model $\left(\mathrm{K}_{\mathrm{A}}\right)$ are compared with the affinities calculated by radioligand binding $\left(\mathrm{K}_{\mathrm{i}}\right)$, the $\mathrm{K}_{\mathrm{A}}$ for dexmedetomidine and octopamine were still 27-fold and 73-fold higher, respectively, than their $K_{i}$, which suggests that sodium must be decreasing the affinity for agonists.

Given the discrepancy between the $\mathrm{K}_{\mathrm{A}}$ and $\mathrm{K}_{\mathrm{i}}$ values for the agonists, we decided to measure the affinity of rauwolscine using the Schild analysis functional approach. Because we had obtained the affinities for rauwolscine in the presence of 15 and $240 \mathrm{mM}$ sodium, it was hypothesized that the agonist affinity calculated by Schild analysis would be similar to the $\mathrm{K}_{\mathrm{i}}$ in the presence of $15 \mathrm{mM}$ sodium (the same concentration as inside mammalian HEK293 cells). However, increasing concentrations of rauwolscine not only reduced dexmedetomidine's potency, but also its efficacy. If rauwolscine was a perfectly competitive antagonist of dexmedetomidine, then dexmedetomidine's potency would be decreased in the presence of increasing concentrations of rauwolscine, but its efficacy would be unaffected. Because dexmedetomidine's efficacy was affected, the results suggest that rauwolscine interacts with the BiOctR at an allosteric site that is coupled to the dexmedetomidine site via a noncompetitive interaction. Taken together, the evidence suggests it is not possible to obtain the true affinity for agonists (in their high-affinity state), and possibly some antagonists as well, when using $\left[{ }^{3} \mathrm{H}\right]$ rauwolscine as the radioligand at BiOctR due to the requirement that sodium be present to measure $\left[{ }^{3} \mathrm{H}\right]$ rauwolscine binding. Furthermore, saturation isotherm-binding experiments on the $\alpha_{2 \mathrm{C}}-\mathrm{AR}$ in the absence or presence of increasing concentrations of dexmedetomidine also demonstrated that $\left[{ }^{3} \mathrm{H}\right]$ rauwolscine and dexmedetomidine are not competing for the same site, i.e., the interaction is a noncompetitive allosterism. This provides molecular mechanistic support for the idea that the $\alpha$-like OctRs and $\alpha$-ARs share a common evolutionary link.

From a physiologic perspective, OctR agonists have been demonstrated to have cyprid antifouling properties (see Introduction). In a previous study, medetomidine was reported to act as a BiOctR agonist and to induce hyperactivity in B. improvisus cyprids (Lind et al., 2010). In this study, octopamine and all of the imidazoles/imidazolines that had full agonist activity at BiOctR were able to induce a hyperactivity response in $B$. amphitrite cyprids. However, the less potent partial agonist, levomedetomidine, was not able to induce a statistically significant hyperactivity response when tested at the same concentrations as the more potent full agonists. Yet, it seems possible that a significant effect might be achieved at a higher concentration of levomedetomidine, as was the case for the considerably less potent endogenous full agonist octopamine, although solubility issues prevented us from testing levomedetomidine at higher concentrations. Overall, we found that structurally diverse compounds with $\mathrm{G}_{\mathrm{q}}$-mediated agonist activity at the BiOctR can induce a hyperactivity response in barnacle cyprids, suggesting that $\mathrm{G}_{\mathrm{q}}$ signaling is a good in vitro measure for evaluating large numbers of compounds to identify those with potential antifouling property against barnacles.

In conclusion, we have demonstrated for the first time that, with some notable differences, the cloned $\alpha$-like barnacle and fruit fly OctRs share a similar functional profile for a wide range of mammalian GPCR agonists and antagonists, and that the BiOctR exhibits sodium sensitivity for both agonists and antagonists. Furthermore, we propose that rauwolscine preferentially binds to an allosteric site on BiOctR that is cooperatively coupled with the sodium binding site and the orthosteric agonist low-affinity state of the receptor.

\section{Acknowledgments}

The authors thank Dr. Ulrika Lind, Department of Marine Sciences, University of Gothenburg, Goteborg, Sweden and Dr. Ronald L. Davis, Department of Neuroscience, The Scripps Research Institute, Jupiter, Florida for generously providing the BiOctR and DmOctR sequences, respectively, and Dr. Myron Toews, Department of Pharmacology \& Experimental Neuroscience, University of Nebraska Medical Center, Omaha, Nebraska for generously providing HEK293 cells stably expressing the human $\alpha_{2 \mathrm{C}}$ adrenergic receptor.

\section{Authorship Contributions \\ Participated in research design: Dalwadi, Schetz. \\ Conducted experiments: Dalwadi. \\ Performed data analysis: Dalwadi, Schetz. \\ Wrote or contributed to the writing of the manuscript: Dalwadi, Schetz.}

\section{References}

Ballesteros JA and Weinstein H (1995) Integrated methods for the construction of three-dimensional models and computational probing of structure-function relations in G protein-coupled receptors, in Receptor Molecular Biology, Methods in Neurosciences (Sealfon SC ed) vol 25, pp 366-428, Academic Press, San Diego. 
Berntsson KM, Jonsson PR, Lejhall M, and Gatenholm P (2000) Analysis of behavioural rejection of micro-textured surfaces and implications for recruitment by the barnacle Balanus improvisus. J Exp Mar Biol Ecol 251:59-83.

Bischof LJ and Enan EE (2004) Cloning, expression and functional analysis of an octopamine receptor from Periplaneta americana. Insect Biochem Mol Biol 34:511-521.

Black JW, Leff P, Shankley NP, and Wood J (2010) An operational model of pharmacological agonism: the effect of $\mathrm{E} /[\mathrm{A}]$ curve shape on agonist dissociation constant estimation. 1985. Br J Pharmacol 160 (Suppl 1):S54-S64.

Blume AJ, Lichtshtein D, and Boone G (1979) Coupling of opiate receptors to adenylate cyclase: requirement for Na+ and GTP. Proc Natl Acad Sci USA 76:5626-5630.

Castille FL and Lawrence AL (1981) A comparison of the osmotic, sodium, and chloride concentrations between the urine and hemolymph of Penaeus setiferus (L.) and penaeus stylirostris stimpson. Comp Biochem Physiol Part A Physiol 70:525-528.

Dahlström M and Elwing H (2006) Adrenoceptor and other pharmacoactive compounds as putative antifoulants. Prog Mol Subcell Biol 42:171-202.

Dahlström M, Mårtensson LGE, Jonsson PR, Arnebrant T, and Elwing H (2000) Surface active adrenoceptor compounds prevent the settlement of cyprid larvae of Balanus improvisus. Biofouling 16:191-203.

Dahms HU, Jin T, and Qian PY (2004) Adrenoceptor compounds prevent the settlement of marine invertebrate larvae: Balanus amphitrite (Cirripedia), Bugula neritina (Bryozoa) and Hydroides elegans (Polychaeta). Biofouling 20:313-321.

Dalwadi DA, Kim S, Amdani SM, Chen Z, Huang RQ, and Schetz JA (2016) Molecular mechanisms of serotonergic action of the HIV-1 antiretroviral efavirenz. Pharmacol Res 110:10-24.

Dereeper A, Guignon V, Blanc G, Audic S, Buffet S, Chevenet F, Dufayard JF, Guindon S, Lefort V, and Lescot M, et al. (2008) Phylogeny.fr: robust phylogenetic analysis for the non-specialist. Nucleic Acids Res 36(Web Server issue):W465-W469.

Dudai Y and Zvi S (1984) High-affinity [3H]octopamine-binding sites in Drosophila melanogaster: interaction with ligands and relationship to octopamine receptors. Comp Biochem Physiol Physiol C 77:145-151.

Enan EE (2005) Molecular and pharmacological analysis of an octopamine receptor from American cockroach and fruit fly in response to plant essential oils. Arch Insect Biochem Physiol 59:161-171.

Ericksen SS, Cummings DF, Weinstein H, and Schetz JA (2009) Ligand selectivity of D2 dopamine receptors is modulated by changes in local dynamics produced by sodium binding. J Pharmacol Exp Ther 328:40-54.

Erspamer V and Boretti G (1951) Identification and characterization, by paper chromatography, of enteramine, octopamine, tyramine, histamine and allied substances in extracts of posterior salivary glands of octopoda and in other tissue extracts of vertebrates and invertebrates. Arch Int Pharmacodyn Ther 88:296-332.

Gerhardt CC, Lodder HC, Vincent M, Bakker RA, Planta RJ, Vreugdenhil E, Kits KS, and van Heerikhuizen H (1997) Cloning and expression of a complementary DNA encoding a molluscan octopamine receptor that couples to chloride channels in HEK293 cells. J Biol Chem 272:6201-6207.

Guindon S and Gascuel O (2003) A simple, fast, and accurate algorithm to estimate large phylogenies by maximum likelihood. Syst Biol 52:696-704.

Han KA, Millar NS, and Davis RL (1998) A novel octopamine receptor with preferential expression in Drosophila mushroom bodies. $J$ Neurosci 18:3650-3658.

Kastner KW, Shoue DA, Estiu GL, Wolford J, Fuerst MF, Markley LD, Izaguirre JA, and McDowell MA (2014) Characterization of the Anopheles gambiae octopamine receptor and discovery of potential agonists and antagonists using a combined computational-experimental approach. Malar $J$ 13:434.
Katritch V, Fenalti G, Abola EE, Roth BL, Cherezov V, and Stevens RC (2014) Allosteric sodium in class A GPCR signaling. Trends Biochem Sci 39:233-244.

Kortagere S, Gmeiner P, Weinstein H, and Schetz JA (2004) Certain 1,4 disubstituted aromatic piperidines and piperazines with extreme selectivity for the dopamine D4 receptor interact with a common receptor microdomain. Mol Pharmacol 66:1491-1499.

Kumar V, Bansal G, Patel J, and Gopi Mohan C (2014) Structure-function prediction of $\alpha 2 \mathrm{~A}-, \alpha 2 \mathrm{~B}-$, and $\alpha 2 \mathrm{C}$-adrenoceptors using homology model assisted antagonist binding study. Med Chem Res 23:735-746.

Leff P, Martin GR, and Morse JM (1985) Application of the operational model of agonism to establish conditions when functional antagonism may be used to estimate agonist dissociation constants. Br J Pharmacol 85:655-663.

Limbird LE, Speck JL, and Smith SK (1982) Sodium ion modulates agonist and antagonist interactions with the human platelet alpha 2-adrenergic receptor in membrane and solubilized preparations. Mol Pharmacol 21:609-617.

Lind U, Alm Rosenblad M, Hasselberg Frank L, Falkbring S, Brive L, Laurila JM Pohjanoksa K, Vuorenpää A, Kukkonen JP, Gunnarsson L, et al. (2010) Octopamine receptors from the barnacle balanus improvisus are activated by the alpha2adrenoceptor agonist medetomidine. Mol Pharmacol 78:237-248.

Malmberg A, Jackson DM, Eriksson A, and Mohell N (1993) Unique binding characteristics of antipsychotic agents interacting with human dopamine D2A, D2B and D3 receptors. Mol Pharmacol 43:749-754.

Nathanson JA (1985) Phenyliminoimidazolidines: characterization of a class of potent agonists of octopamine-sensitive adenylate cyclase and their use in understanding the pharmacology of octopamine receptors. Mol Pharmacol 28: $254-268$.

Neve KA (1991) Regulation of dopamine D2 receptors by sodium and pH. Mol Pharmacol 39:570-578.

Neve KA, Cumbay MG, Thompson KR, Yang R, Buck DC, Watts VJ, DuRand CJ, and Teeter MM (2001) Modeling and mutational analysis of a putative sodiumbinding pocket on the dopamine D2 receptor. Mol Pharmacol 60:373-381.

Roeder T (1995) Pharmacology of the octopamine receptor from locust central nervous tissue (OAR3). Br J Pharmacol 114:210-216.

Roeder T (1999) Octopamine in invertebrates. Prog Neurobiol 59:533-561.

Schetz JA (2005) Allosteric modulation of dopamine receptors. Mini Rev Med Chem $\mathbf{5}$ : $555-561$.

Schetz JA, Chu A, and Sibley DR (1999) Zinc modulates antagonist interactions with D2-like dopamine receptors through distinct molecular mechanisms. J Pharmacol Exp Ther 289:956-964.

Schetz JA and Sibley DR (2001) The binding-site crevice of the D4 dopamine receptor is coupled to three distinct sites of allosteric modulation. J Pharmacol Exp Ther 296:359-363.

Selley DE, Cao CC, Liu Q, and Childers SR (2000) Effects of sodium on agonist efficacy for G-protein activation in mu-opioid receptor-transfected $\mathrm{CHO}$ cells and rat thalamus. Br J Pharmacol 130:987-996.

Address correspondence to: Dr. John A. Schetz, University of North Texas Health Science Center, Department of Pharmacology and Neuroscience, Center for Neuroscience Discovery/Institute for Healthy Aging, 3500 Camp Bowie Boulevard, Fort Worth, TX 76107. E-mail: John.Schetz@unthsc.edu 